\title{
12
}

\section{Electro-Optical Scanners}

\section{Timothy K. Deis}

Consultant

Pittsburgh, Pennsylvania, USA

\section{Daniel D. Stancil}

Carnegie Mellon University

Pittsburgh, Pennsylvania, USA

\section{Carl E. Conti}

Consultant

Hammondsport, New York, USA

\section{CONTENTS}

12.1 Introduction

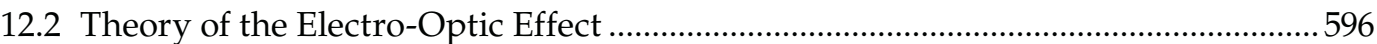

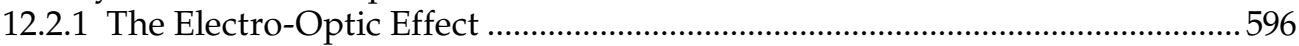

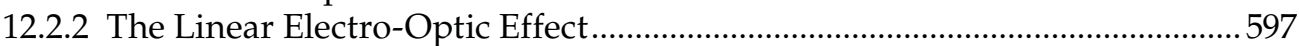

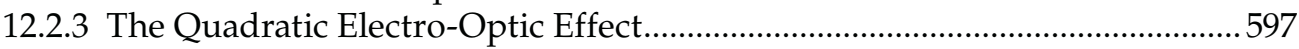

12.3 Principal Types of Electro-Optic Deflectors .............................................................598

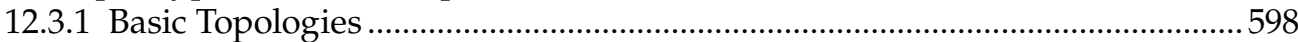

12.3.2 Terminology for Describing Electro-Optic Scanners ......................................598

12.3.2.1 Beam Displacement and Deflection Angle........................................598

12.3.2.2 Pivot Point ................................................................................................59

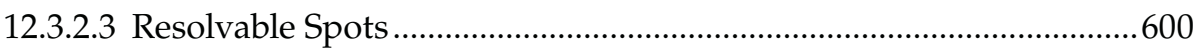

12.3.3 Single Elements and Assemblies of Single Elements .......................................601

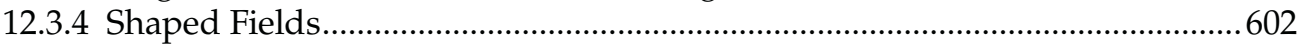

12.3.4.1 Graded Index with Uniform Applied Voltage ....................................603

12.3.4.2 Graded Index with Constant Spacing ...............................................605

12.3.4.3 Graded Index with Constant Spacing and Single Voltage ................606

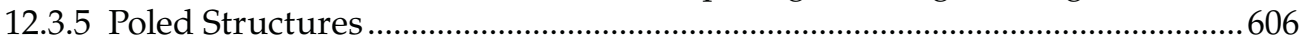

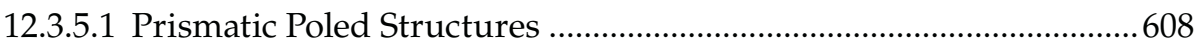

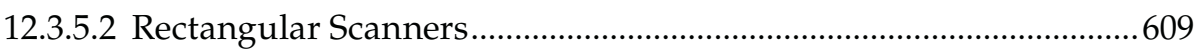

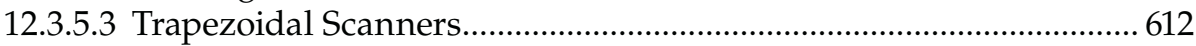

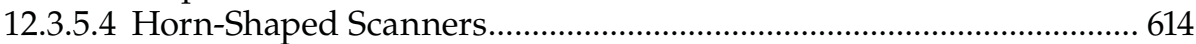

12.3.5.5 Domain Inverted Total Internal Reflection Deflectors ..................... 617

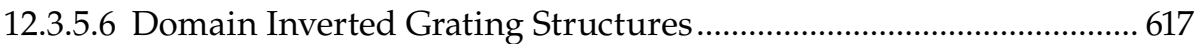

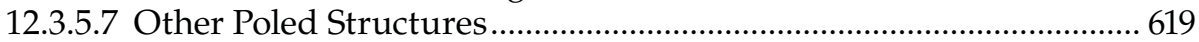

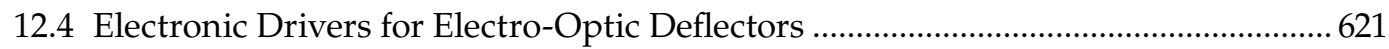

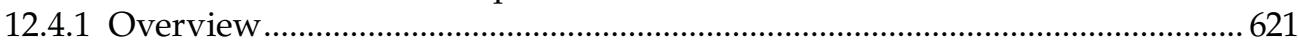

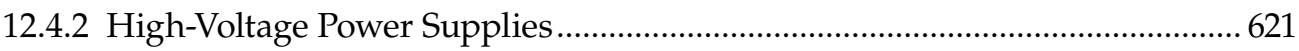

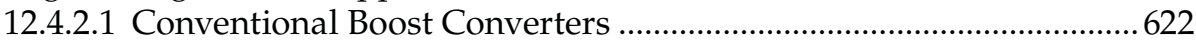

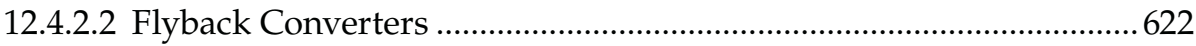




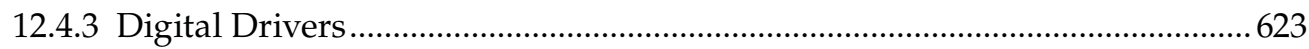

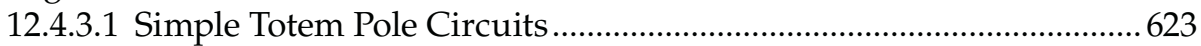

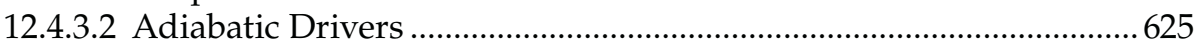

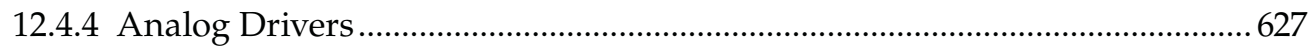

12.5 Properties and Selection of Electro-Optic Materials ..............................................628

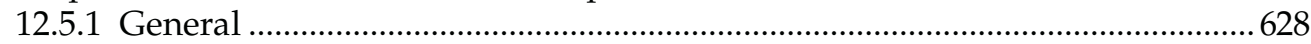

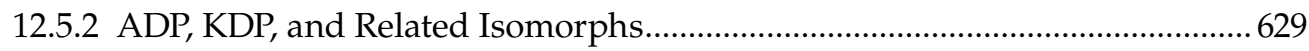

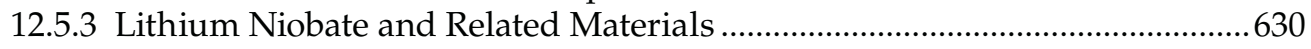

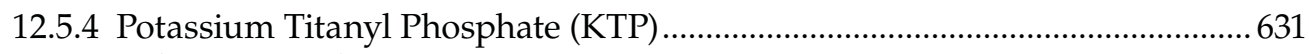

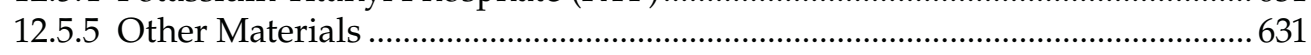

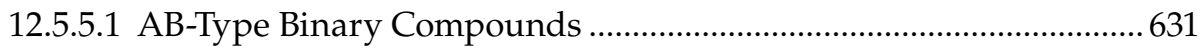

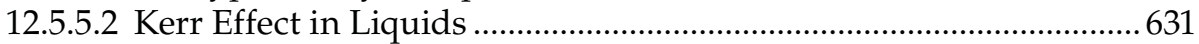

12.5.5.3 Electro-Optic Ceramics in the $(\mathrm{Pb}, \mathrm{La})(\mathrm{Zr}, \mathrm{Ti}) \mathrm{O}_{3}$ System...................6 631

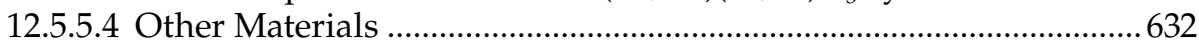

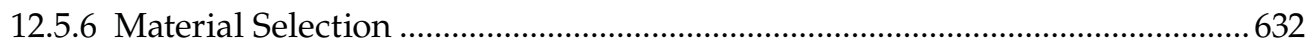

12.6 Electro-Optic Deflection System Design Process....................................................633

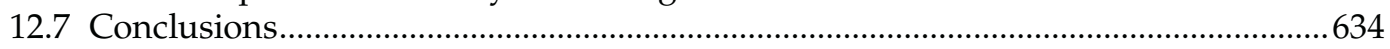

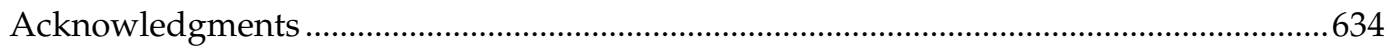

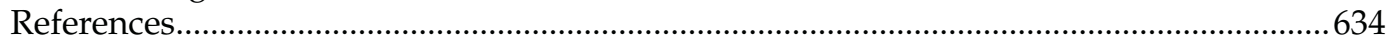

\subsection{INTRODUCTION}

Electro-optic deflection systems are most typically considered in applications where high deflection speed is the paramount selection criteria. They can also have other attractive features, such as high optical efficiency and physical robustness, but these are usually secondary concerns after deflection speeds that can exceed $10^{9} \mathrm{rad} / \mathrm{s}$.

Requirements for great speeds are driven by advancements in two technical areas: lasers and computing. Progress in laser technology has resulted in reliable, low-cost compact sources with high power. Many applications, such as material marking, require a certain amount of energy to be delivered and are somewhat independent of the time period that the energy is delivered over. More powerful lasers can deliver the required energy dose in a short period of time, putting pressure on deflection system designers for higher operating speeds.

Advances in computing and communications have resulted in ever-higher data rates delivered to the laser deflection system. Data rates in applications such as displays now far surpass the control bandwidth of most mechanical systems such as galvanometers, piezoelectric deflectors, and microelectromechanical systems (MEMS) mirrors, which are limited by mechanical inertia effects. Relatively low bandwidth, usually less than $10 \mathrm{kHz}$, makes these systems a poor choice if a laser or other light source can deliver the required energy dose within a time period that matches the desired data rate, which often exceeds $\mathrm{MHz}$ speeds. In the realm of electrical circuits, electro-optic (EO) deflectors are essentially capacitors, which can be charged very quickly with appropriate driver circuitry and can operate at $\mathrm{MHz}$ speeds with ease.

The most common technical competition to EO deflection is acousto-optic (AO) deflection, covered elsewhere in this volume. Electro-optic systems are often faster and more 
optically efficient than $\mathrm{AO}$ systems. They often can handle higher beam powers than $\mathrm{AO}$ systems because there is no need to achieve a tight beam focus in the device for maximum speeds. The AO systems can be designed to have much higher resolutions, offering hundreds or thousands of resolvable spots compared to 5 to 100 that are more typical of EO systems. Both EO scanners and many AO scanners have polarization-dependent characteristics, which can limit their application. In general, the crystal and glass materials for $\mathrm{AO}$ devices are more readily available and have uniform high quality, unlike the materials for EO deflectors, which can be hard to procure or may have uncertain property characteristics. Electronic drivers for $\mathrm{AO}$ deflection systems usually require higher input power than those for EO systems.

Examples of current applications where the combination of high-power compact laser sources and high-speed data delivery are driving the evaluation or adoption of EO deflection systems include: encoding image data onto printing plates in plate setting machines, controlling optical paths in communications switching systems, creating jitter-free laser projection displays, and maintaining alignment in free space communications systems over long distances.

Electro-optic deflectors or scanners can be configured to meet a variety of requirements. Choices of the optical material, typically a crystal, the drive electronics, and optical path can be made to address all of the following functional requirements:

1. Error correction: high-speed $\left(10^{6} \mathrm{rad} / \mathrm{s}\right)$ and low deflection (order of milli-rad) characteristics can be used to provide facet-to-facet error correction of polygon scanners. The same characteristics can also be used to "debounce" a galvanometer-based display system.

2. Switching: high speed ( $<100$ ns steps) and low deflection (order of 10 resolution spots) can be used to switch collimated light in fiber optic switches, optical backplanes, and optical computers.

3. Modulation: in cases where it is not possible to directly modulate a laser, as with some diode-pumped sources, a deflector can be used to modulate the beam by deflecting it toward or past a beam stop. Applications of this sort include displays, printing plate production, and marking.

There is a class of EO device that is sometimes confused with deflectors or scanners. This class uses the Kerr effect effectively to rotate the polarization of light traversing the device. Such devices can be used as modulators for polarized light sources by rotating the plane of polarization to be parallel or perpendicular to a polarizer downstream in the system. Such systems are commercially available, and have been used in some printing applications.

If a polarization-dependent mirror or beam splitter is used in place of the polarizer in the above system, the beam may be switched to one of two output positions by electrooptically controlling the polarization of the beam. These devices may be cascaded to produce many output positions. These and related systems are not the focus of the current text and will not be covered further.

The present effort builds on the previous edition, authored by Clive L.M. Ireland and John Martin Ley. ${ }^{1}$ Much material has been carried over, especially that related to materials properties and basic physics. New material has been added to cover domain-inverted scanner designs, which have recently been applied in commercial products. A new section was added to address some of the options and characteristics of electronic drivers suitable 
for commercial applications. Experience has shown that this part of the system can be as difficult to realize as the optical elements. As with the material relating to optical materials and designs, the electronic driver material is intended to serve as a general reference for guiding development efforts, not as a definitive treatise on the subject.

\subsection{THEORY OF THE ELECTRO-OPTIC EFFECT}

\subsubsection{The Electro-Optic Effect}

The EO deflectors and scanners discussed below all rely on the EO effect evidenced to some degree by all materials. Only a few materials exhibit property changes with an applied electric field large enough to be exploited for use in deflecting and switching applications. The index of refraction of these materials, typically crystals, changes when an electric field is applied to such a degree that it can be used to usefully deflect a beam according to the rules of normal refractive optics.

In crystals, the direction of the polarization induced by an applied electric field may differ in direction from that of the applied field. Mathematically, this means that the relative permittivity must be represented by a second-rank tensor:

$$
D_{i}=e_{0} k_{i j} E_{j}=e_{0} E_{i}+P_{i}
$$

where $\varepsilon_{0}$ is the permittivity of free space, $\kappa_{i j}$ is the relative dielectric tensor, and $E_{i}$ and $P_{t}$ are the $i$ th components of the electric field and the induced polarization, respectively, and summation over repeated indices is assumed. We will restrict our discussion to crystals that are essentially neither magnetic nor optically active, and that exhibit negligible absorption. In this case, $\kappa_{i j}$ is a real, symmetric tensor.

A convenient geometric representation of any symmetric second-rank tensor $S_{i j}$ is an ellipsoidal or hyperboloidal surface defined by

$$
S_{i j} x_{i} x_{j}=1
$$

Thus we can construct such a surface for $\kappa_{i j}$, or its inverse $(1 / \kappa)_{i j}$. In contrast, the refractive index-given by the square root of the relative permittivity in isotropic materials-does not transform as a second-rank tensor. Since $\kappa=n^{2}$ in an isotropic material, it is conventional to adopt the following notation:

$$
\left(\frac{1}{n^{2}}\right)_{i j} x_{i} x_{j}=1
$$

This ellipsoidal surface is called the index ellipsoid, or indicatrix. In the coordinate system in which $\left(1 / n^{2}\right)_{i j}$ is diagonal, Equation 12.3 reduces to

$$
\frac{x^{2}}{n_{x}^{2}}+\frac{y^{2}}{n_{y}^{2}}+\frac{z^{2}}{n_{z}^{2}}=1
$$

This surface has a simple geometric interpretation. The principal axes of the ellipsoid correspond to directions in the crystal for which $D$ is parallel to $E$, and the refractive indices for waves polarized along these directions are $n_{x}, n_{y}$, and $n_{z}$. 


\subsubsection{The Linear Electro-Optic Effect}

The crystal materials commonly used for EO devices do not possess inversion symmetry (see Stancil ${ }^{2}$ for a complete explication of the tensor properties of crystals), meaning that the application of an electric field induces a small change in the refractive index that is proportional to the field, and reverses in sign when the field reverses. This is known as Pockel's Effect, or the Linear Electro-Optic Effect.

In the presence of a uniform electric field, the changes in the indices of refraction of such materials can be shown to be

$$
\Delta\left(\frac{1}{n^{2}}\right)_{i j}=r_{i j, k} E_{k}
$$

where $r_{i j, k}$ is the linear EO tensor, whose values are readily available from data published in the literature and from crystal suppliers.

As an example, consider the EO effect in $\mathrm{KH}_{2} \mathrm{PO}_{4}$, also known as KDP. In this crystal (and all crystals with symmetry $\overline{4} 2 \mathrm{~m}$ ) the only nonzero components of the EO tensor are $r_{41}=r_{52}$, and $r_{63}$. For simplicity, we will consider the case of a static electric field applied along the optic axis, $E_{3}$. For further simplicity, for small $\Delta n$, which is normally the case, $\Delta\left(1 / n^{2}\right)=-2 \Delta n / n^{3}$. Thus, the refractive index seen by an optical wave polarized along the $\langle 110\rangle$ direction is approximately given by

$$
n_{1}=n_{o}-\frac{1}{2} n_{o}^{3} r_{63} E_{3}
$$

where use has been made of the fact that $r_{63} E_{3} 1 / n_{o}^{2}$. Similarly, the index along the $\langle 1 \overline{1} 0\rangle$ direction is

$$
n_{2}=n_{o}+\frac{1}{2} n_{o}^{3} r_{63} E_{3}
$$

The index along the optical axis $\langle 001\rangle$ is unchanged $\left(n_{3}=n_{e}\right)$.

Note that the index change observed depends on the polarization of the light transiting the region with the applied field. This effect makes the performance of many EO scanners "polarization dependent," where the deflection achieved depends on the polarization of the beam. This can result in splitting randomly polarized beams and is the reason that most EO scanners are used with polarized beams only.

\subsubsection{The Quadratic Electro-Optic Effect}

Refractive index changes proportional to the square of the applied field are permitted by symmetry in all materials. Besides crystals such as $\mathrm{KDP}$ and $\mathrm{LiNbO}_{3}$, liquids that are strongly polar are of particular EO interest since they can exhibit a high anisotropic, optic polarizability. By applying a strong external field, the molecules of these substances partially align with the field, causing the bulk material to become biréfringent.

The component of a beam polarized parallel to the main polarizability of the molecules, usually nearly parallel to the dipole moment of the molecule, sees an increase in refractive index relative to that of the orthogonal polarization. This effect, which was observed by Kerr in glass and other materials, is generally described by the following equation:

$$
n_{\mathrm{p}}-n_{\mathrm{s}}=B 1 E^{2}
$$


Here $\lambda$ is the vacuum wavelength of the beam, $B$ is the Kerr constant for the material, and $n_{\mathrm{p}}$ and $n_{\mathrm{s}}$ are the parallel and orthogonal refractive index components, respectively, and $E$ is the applied electric field.

A variety of Kerr materials and devices have been studied in the past, see Lee and Hauser $^{3}$ and Kruger et al. ${ }^{4}$ At the time of this writing, late 2002, there do not appear to be any commercial devices or systems based on the quadratic EO effect in production.

\subsection{PRINCIPAL TYPES OF ELECTRO-OPTIC DEFLECTORS}

\subsubsection{Basic Topologies}

The EO effect can be utilized in a variety of basic ways, with details seemingly limited only by the imagination of very clever practitioners. The design problem is one of selecting a gross geometry that the beam can traverse, and then selecting the geometry and magnitude of desired index change. To provide some order, the following nomenclature is used:

1. Shaping of gross geometry: at its simplest, an EO scanner can consist of a prismatic crystal element with electrodes covering both ends. As the potential across the element is changed, it acts like an electrically controlled prism.

2. Electrically shaped fields: When a bulk prism would be too small for easy handling, or if the field strength desired would result in electrical breakdowns across the side surfaces, electrical fields may be shaped. This is normally achieved by using shaped electrodes of constant potential. A less common approach is to use electrodes with finite or graded conductivity so that a varying voltage profile is obtained when a current is applied.

3. Poled structures: some EO materials, such as $\mathrm{LiNbO}_{3}$ and $\mathrm{LiTaO}_{3}$, may be "poled," a process that can result in geometrically precise crystalline domains within the bulk material. A uniform electric field applied to poled structures will result in equal and opposite changes in index within each domain according to its orientation.

\subsubsection{Terminology for Describing Electro-Optic Scanners}

Electro-optic scanners can be discussed using much of the same terminology as other types of scanners. The difficult trade-offs between size, voltage, and material properties create some nuances that are important to consider.

\subsubsection{Beam Displacement and Deflection Angle}

A schematic EO scanner is shown in Figure 12.1. In the presence of a linear index variation (constant gradient) across the width of the scanner, the trajectory of the center of the beam within the scanner is described by the parabolic relation ${ }^{5}$

$$
X(z)=\frac{1}{n} \frac{\mathrm{d} n}{\mathrm{~d} x} \frac{z^{2}}{2} \approx \frac{1}{2} \frac{\Delta n}{n} \frac{z^{2}}{W}
$$




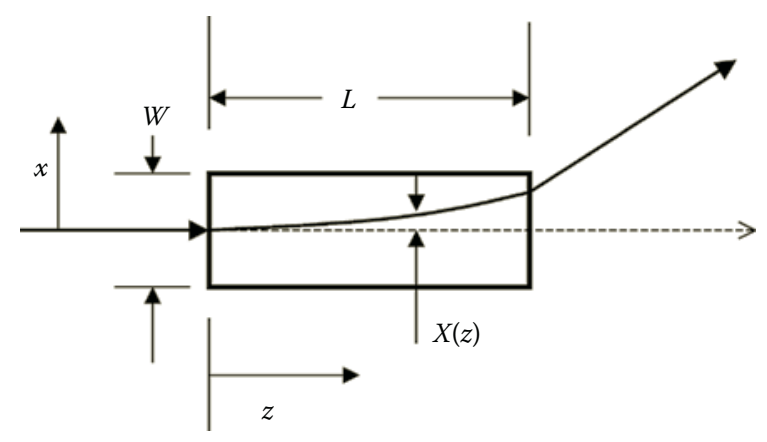

FIGURE 12.1

Schematic electro-optical scanner of width $W$ and length $L$. An index of refraction profile of the form $n(x)=n_{0}+$ $k x$ is assumed. The optical beam to be deflected enters from the left.

where $X(z)$ is the displacement of the beam center from the optical axis at position $z, \Delta n$ is the total change in index across the scanner, $n$ is the nominal index of refraction (in the absence of an EO shift), and $W$ is the width of the scanner.

The deflection angle at a particular position $z$ is given by the slope of the trajectory at that point, or the derivative of Equation 12.9:5,6

$$
q_{\text {in }}(z)=\frac{1}{n} \frac{\mathrm{d} n}{\mathrm{~d} x} z \approx \frac{\Delta n}{n} \frac{z}{W}
$$

When the beam exits the material, the angle is increased by the factor $n$, owing to the small-angle form of Snell's Law. The external deflection angle for the scanner is therefore obtained by evaluating Equation 12.10 at $z=L$ and multiplying by $n$ :

$$
q_{\mathrm{def}}=\frac{\mathrm{d} n}{\mathrm{~d} x} L \approx \Delta n \frac{L}{W}
$$

The displacement of the beam at the output facet of the scanner is finally given by

$$
d=\frac{1}{2} \frac{\Delta n}{n} \frac{L^{2}}{W}
$$

\subsubsection{Pivot Point}

Comparison of Equations 12.12 and 12.10 shows that the output displacement can also be expressed as

$$
d=\frac{1}{2} q_{\text {in }} L
$$

This suggests that although the actual trajectory is parabolic, the output angle and displacement are correctly given by assuming that the beam has an abrupt deflection of $\theta_{\text {in }}$ a distance of $L / 2$ from the output plane. ${ }^{7}$ We call this the pivot point, and define it more generally as

$$
L_{\mathrm{P}, \mathrm{in}}=\frac{X(L)}{\mathrm{q}_{\mathrm{in}}(L)}
$$


A scanner has a well-defined pivot point when $L_{\mathrm{P}, \text { in }}$ does not depend on the magnitude of the index variation $\Delta n$.

When the beam deflection is viewed outside the scanner, the deflection also appears to have a pivot point, although displaced, just as an object on the bottom of a swimming pool appears to be displaced when viewed from outside the pool. From outside the scanner, the pivot point appears to be a distance $L_{\mathrm{P}}$ from the output plane:

$$
L_{\mathrm{P}}=\frac{X(L)}{q_{\text {def }}(L)}=\frac{L_{\mathrm{P}, \text { in }}}{n}
$$

The existence of a pivot point is significant for the design of optical systems containing EO scanners. From the optical system point of view, the scanner can be represented simply by a mirror at a distance $L_{\mathrm{P}}$ from the output plane that introduces the deflection $\theta_{\text {def }}$.

\subsubsection{Resolvable Spots}

Perhaps the best way to compare various scanner technologies is to use the number of "resolvable spots." The deflection angle can be magnified or reduced with other optical elements, but the number of resolvable spots will remain constant. The number of resolvable spots is given by the number of beam diameters corresponding to the lateral displacement at a certain distance, usually the far field. Clearly this number depends on the way that the beam diameter is defined. For our purposes, we will assume the beam is well described by a fundamental Gaussian beam whose $1 / e^{2}$ intensity radius is given by the Gaussian beam waist $w(z)$ :

$$
w(z)=w_{0} \sqrt{1+\left(\frac{I\left(z-z_{0}\right)}{p w_{0}^{2}}\right)^{2}}
$$

where $w_{0}$ is the minimum radius at the beam waist, $\lambda$ is the optical wavelength, and $z_{0}$ is the location of the minimum radius, or waist. The number of resolvable spots is given by

$$
N_{\mathrm{U}}(z)=\frac{S(z)}{2 w(z)}+1
$$

where $N_{\mathrm{U}}$ is the number of spots assuming unipolar deflection (deflection only to one side of the optical axis) and $S$ is the displacement of the beam at the observation plane (see Figure 12.2). Often scanners are used with bipolar drive voltages, resulting in a total beam displacement of $2 S$. Thus the number of bipolar resolvable spots is

$$
N_{\mathrm{B}}(z)=\frac{S(z)}{w(z)}+1
$$

The displacement $S$ at a distance $z$ from the pivot point is given by

$$
S=q_{\mathrm{def}} z
$$



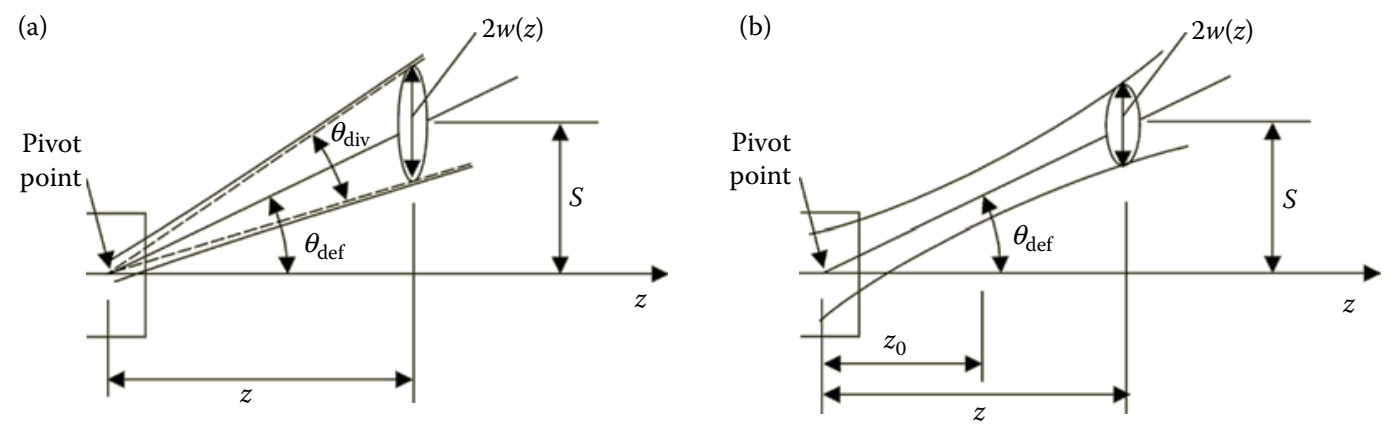

FIGURE 12.2

Geometry illustrating the concept of resolvable spots: (a) beam focus/waist near the pivot point of the scanner, and (b) beam focus/waist beyond the output.

It is instructive to consider the case with the waist collocated with the pivot point $\left(z_{0}=\right.$ 0 ) and the observation point arbitrarily far away. This limit results in the number of spots in the far field:

$$
\begin{gathered}
N_{\mathrm{U}, \mathrm{FF}} \approx \frac{q_{\mathrm{def}} p w_{0}}{2 I}+1=\frac{q_{\mathrm{def}}}{q_{\mathrm{div}}}+1 \\
N_{\mathrm{B}, \mathrm{FF}} \approx \frac{2 q_{\mathrm{def}}}{q_{\mathrm{div}}}+1
\end{gathered}
$$

where

$$
q_{\text {div }}=\frac{21}{p w w_{0}}
$$

is the far-field divergence (full angle) of the Gaussian beam. The displacement clearly gets larger the further away the observation plane is, but this does not result in an increase in spots since the beam divergence causes the spot diameter to increase at the same rate in the far field.

It is important to note that the maximum number of resolvable spots is achieved only in the far field. Many practical EO systems, such as deflection-based modulators, do not require operation in the far field. It is critically important to perform accurate simulations and ray tracings as part of the design process.

Other definitions of beam diameter are sometimes used, depending on the application. For instance, flat-top power profiles are useful for some laser machining operations. The effect of the diameter definition, and the correspondingly correct description of beam divergence, should be considered when discussing the number of resolvable spots that a particular system may exhibit.

\subsubsection{Single Elements and Assemblies of Single Elements}

One of the most basic optical elements is a prism. Accordingly, one of the most basic EO elements is an electrically controlled prism (Figure 12.3). 


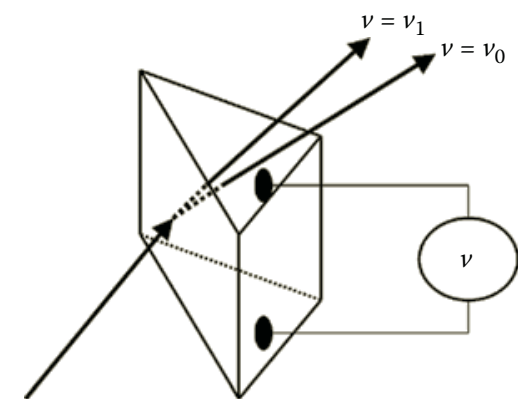

FIGURE 12.3

The simplest electro-optical scanner: a prism fabricated from an electro-optic material having electrodes at each end. As the voltage is varied, the angle of the exiting beam is changed.

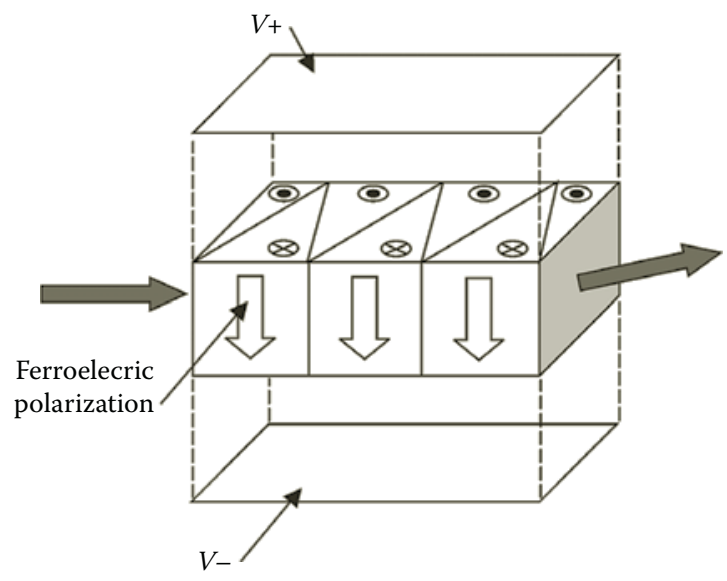

\section{FIGURE 12.4}

Electro-optic prism scanner made from alternating discrete prisms. From Lotspeich, J.F. Electrooptic light-beam deflection. IEEE Spectrum 1968, 5, February, 45-52. With permission.

In practice, the actual deflection about the mean is very small. For example, an equilateral triangle prism fabricated from lithium niobate and operated to $\pm 1 \mathrm{kV} / \mathrm{mm}$ will undergo an index change of approximately \pm 0.0002 , or $0.01 \%$. This change is actually smaller than the accuracy bounds typically quoted by commercial crystal manufacturers for property values.

As discussed previously, the sign of the refractive index change reverses with the direction of polarization (usually coincident with the optic axis of the crystal). Thus a direct extension of the single prism implementation is to assemble several discrete prisms with alternating polarization as shown in Figure 12.4. With proper choice of optical polarization, crystal, and orientation, a voltage applied between conducting electrodes on opposing transverse sides of the assembly increases and decreases the index in alternating prisms generating the $\Delta n$ required for scanner operation. The major disadvantage of this type of construction is the labor-intensive cutting, polishing, and assembly of the prisms.

\subsubsection{Shaped Fields}

An analysis of the energized multi-element assembly shows that it can be accurately represented by a band of material with linearly varying index of refraction. ${ }^{5}$ This leads to 
two interesting design realizations: graded electrical fields with uniform crystal structure, covered in this section, and uniform electrical field and graded crystal structures created through domain inversion, covered in the following section.

\subsubsection{Graded Index with Uniform Applied Voltage}

One way to create a linearly varying spatial index profile is to apply a linearly varying electric field to an EO crystal. Most electrical circuits, especially those designed for lowpower operation, are designed to provide a single voltage across an electrode. However, if the geometric spacing between electrodes is not fixed to a single value, the electrical field between the electrodes will exhibit a spatial variation. If this variation is linear where the beam transits the crystal, a good scanner can be produced. A quadrupole field as shown in Figure 12.5 has the desired behavior near the origin..$^{89}$ If the electrodes are shaped to follow the hyperbolae

$$
x y= \pm \frac{R_{0}^{2}}{2}
$$

then the potential in the region between the electrodes is given by

$$
V=\frac{V_{0}}{R_{0}^{2}} x y
$$

The electric field is obtained by taking the gradient of the potential:

$$
\begin{aligned}
& E_{x}=-\frac{\partial V}{\partial x}=-\frac{V_{0}}{R_{0}^{2}} y \\
& E_{y}=-\frac{\partial V}{\partial y}=-\frac{V_{0}}{R_{0}^{2}} x
\end{aligned}
$$

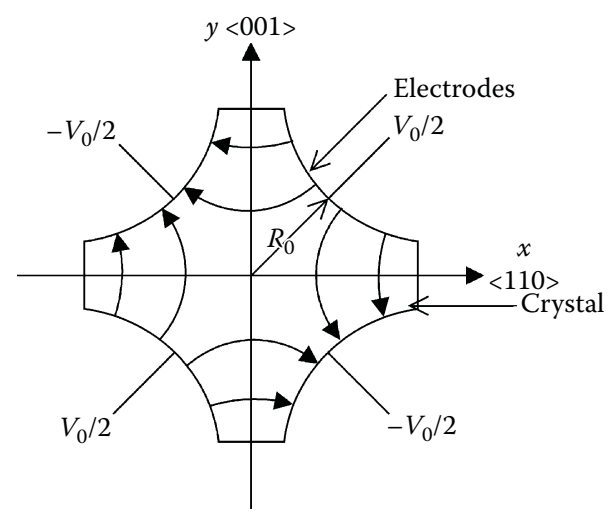

FIGURE 12.5

Geometry for generating a linear electric field profile using quadrupole electrodes in KDP-type materials. Crystallographic directions for proper deflector operation are also shown. Optical beam propagation is perpendicular to the page, and the optical electric field polarization is parallel to the $x$-axis ( $<110>$ direction). (From Fowler, V.J.; Buhrer, CF.; Bloom, L.R. Electro-optic light beam deflector. Proc. IEEE 1964, 52(2), 193-194. With permission.) 
Thus we see that both components of the electric field vary linearly with position. However, if a crystal with symmetry $\overline{4} 2 m$ is oriented as indicated in Figure 12.5 , then there is no EO effect for $E_{x}$, to the first order. Using the index expressions in Table 12.1 for KDP, the index gradient for an optical wave polarized along the $x$-direction $(\langle 110\rangle)$ becomes

$$
\frac{\mathrm{d} n}{\mathrm{~d} x}=\frac{n^{3} r_{63} V_{0}}{2 R_{0}^{2}}
$$

The beam displacement and deflection angle at the scanner output can be obtained by substituting Equation 12.26 into Equations 12.1 and 12.3. The results are

$$
\begin{aligned}
& X(L)=\frac{n^{2} r_{63} V_{0}}{2 R_{0}^{2}} \frac{L^{2}}{2} \\
& q_{\text {def }}=\left(\frac{L n^{3} r_{63}}{2 R_{0}^{2}}\right) V_{0}
\end{aligned}
$$

The deflection sensitivity and pivot point are readily found to be

$$
\begin{gathered}
\frac{q_{\mathrm{def}}}{V}=\frac{L n^{3} r_{63}}{2 R_{0}^{2}} \\
L_{\mathrm{P}, \mathrm{in}}=\frac{L}{2}
\end{gathered}
$$

It is a difficult task to shape a crystal to accommodate hyperbolic electrodes as shown in Figure 12.5. Instead, Figure 12.6a shows a more practical geometry. ${ }^{10}$ The field inside the crystal has been computed using the Finite Element Method ${ }^{11}$ for KDP. Although the electrodes are not shaped precisely like hyperbolae, the field near the center of the crystal is still approximately linear, as shown in Figure 12.6b. Another approximation

\section{TABLE 12.1}

Eigenvalues and Eigenvectors for KDP with an Electric Field Applied Along the Optic Axis ${ }^{a}$

\begin{tabular}{llll}
\hline Eigenvalue & Eigenvector & Index & \multicolumn{1}{c}{$\Delta n$} \\
\hline$\frac{1}{n_{o}^{2}} r_{63} E_{3}$ & $<110>$ & $n_{o}-\frac{\Delta n}{2}$ & $n_{o}^{3} r_{63} E_{3}$ \\
$\frac{1}{n_{o}^{2}}-r_{63} E_{3}$ & $\langle 1 \overline{1} 0\rangle$ & $n_{o}+\frac{\Delta n}{2}$ & $n_{o}^{3} r_{63} E_{3}$ \\
$\frac{1}{n_{e}^{2}}$ & $<001>$ & & 0
\end{tabular}

a For consistency with subsequent sections, $\Delta n$ is the index change between two regions with oppositely oriented field; thus the index change arising from applying a field with a single polarity to a single domain material is $\Delta n / 2$. 
(a)

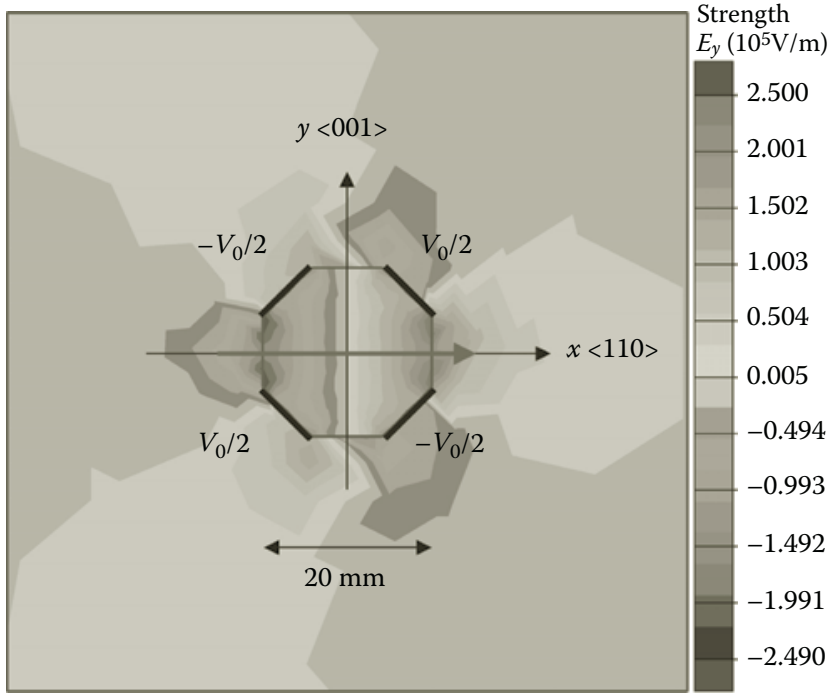

(b)

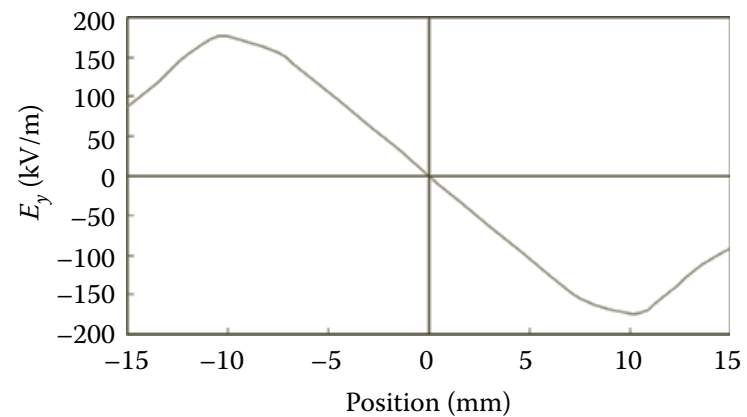

FIGURE 12.6

(a) Finite element analysis ${ }^{11}$ of the electric field in a KDP crystal shaped like an octagon to approximate a quadrupolar field. The shading indicates the strength of the $y$ component of the electric field. The vertical stripes near the center indicate that $E_{y}$ is approximately linear with $x$ and independent of $y$; (b) Calculation of the electric field $E_{y}$ along the contour shown in part (a). Note the linearity of the field near the origin.

of hyperbolic electrodes was developed by Ireland and Ley. ${ }^{12}$ In this case, cylindrical electrodes were used.

\subsubsection{Graded Index with Constant Spacing}

A second option for producing a linearly graded index of refraction depends on depositing a resistive electrode and a conductive electrode on opposite faces of a slab of EO material, as shown in Figure 12.7. When the voltage is applied at the leads, current flows across the resistive electrode, which is essentially a resistor, resulting in a graded electrical field within the device. The change in optical index, which is proportional to the electric field in the material, is thus graded according to the voltage.

The difficulties of producing this type of electrode have limited their consideration for use to very thin devices, on the order of tens of microns. The optical beams are not typically round in these scanners, since a relatively wide stripe is needed for the voltage gradient 


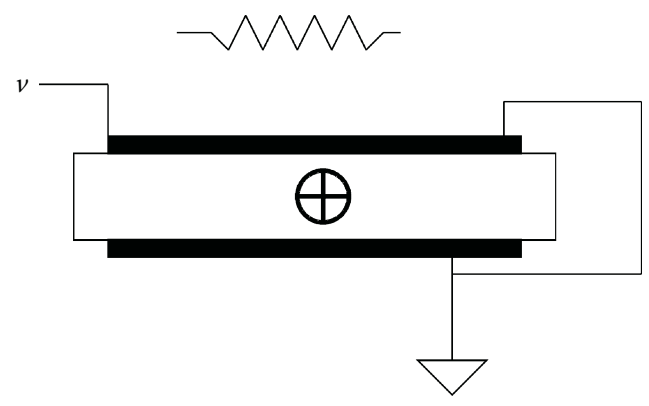

FIGURE 12.7

A resistive electrode may be used to produce graded electrical fields. This is a schematic view, seen end on, of such a device.

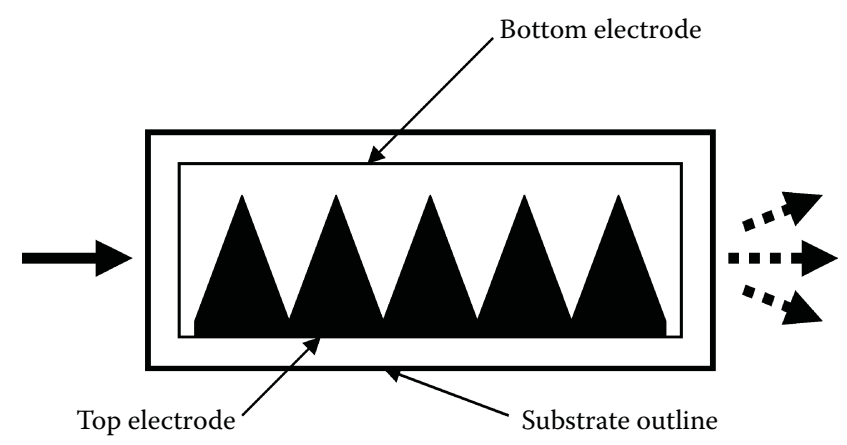

FIGURE 12.8

A serrated electrode, all of which is held at a single voltage, has the effect of producing a graded electrical field over the length of the electro-optical device.

electrodes. Difficulties of coupling optical beams into such wide and thin layers, and their great divergence on the output side add to the difficulties of this approach, although it has been proposed for optical switching for telecommunications use. Electrical heating due to the current flow is another limiting factor.

\subsubsection{Graded Index with Constant Spacing and Single Voltage}

A third approach to producing a gradient in the index of refraction is illustrated in Figure 12.8. The portion of the beam that travels under the root of the conducting top electrode will traverse more material affected by the electrical field than a ray traversing only the tips. This technique suffers from the complex fringing electrical fields around the top electrode. These fields give rise to out-of-plane distortion and other beam quality problems. A wide variety of electrode shapes and spacings are possible, ${ }^{1}$ some of which mitigate the effects of fringing fields to a degree.

\subsubsection{Poled Structures}

There are two ways of achieving an effective linear gradation in index: grading the electric field, or grading the material properties. The techniques covered above, except the 
use of multiple discrete inverted prisms, all use device or electrode geometry to effectively grade the electrical field. Grading the material properties is possible using the technique of "poling" or "domain inversion." This process can be performed completely independently of the base material production, making it an effective tool for device fabrication.

One salient advantage of poled devices is that they are not typically affected by electrical fringing fields. They are normally constructed with cover electrodes that are large enough to ensure uniform fields over the active region of the device, which may contain 50 or more interfaces. The result is that the beam will have to pass through only two fringing fields, at the entrance and exit, versus 100 or more in some field-graded devices. The poling fabrication technique is an outgrowth of research directed toward making quasiphase-matched second harmonic generating gratings. ${ }^{13-17}$ It was discovered that by using photolithographic techniques, domain patterns with virtually any shape can be realized in materials such as $z$-cut lithium niobate, lithium tantalate, and potassium titanyl phosphate and its isomorphs such as rubidium titanyl arsenate.

The basic process includes the following steps:

1. Patterning: photolithographic techniques are used to pattern photoresist on one or both of the crystal surfaces with the shape of the final inverted region. Wafers are often used for greatest compatibility with standard semiconductor processing equipment.

2. Apply poling electrodes: either metal or liquid electrodes are placed on the wafer surface.

3. Poling: high electric fields, greater than the coercive field of the medium or on the order of $20 \mathrm{kV} / \mathrm{mm}$ for lithium niobate, are applied. Practitioners use voltage pulses, ramps, and quasi-DC waveforms - there does not appear to be an accepted standard practice at this time.

4. Apply operating electrodes: this is often done using standard photolithography and thin film deposition. The operating electrodes are typically much larger than the poling electrodes to ensure uniform electric field in the poled region, but they may be limited in size due to the desire to minimize the device's electrical capacitance and therefore driver power requirements.

5. Annealing: temperatures ranging from about $200{ }^{\circ} \mathrm{C}$ to over $1000{ }^{\circ} \mathrm{C}$ are used, with the appropriate temperature and cycle profile determined by experimentation.

During the "poling" step, atoms are shifted in the crystal lattice, but only in the volumes defined by the photolithographically applied patterns. This effectively "flips" the ferroelectric domains without having to cut, polish, AR coat, and reassemble the crystal.

Using electric field poling techniques, it is possible to drive the inverted domain regions completely through relatively thick substrates - up to $3 \mathrm{~mm}$ have been reported for devices fabricated with profile precision of a few microns in RTA (R. Stolzenberger, personal communication, 2003). For more details on the physics of the domain inversion process, see Gopalan et al. ${ }^{18}$ Using such a process, confinement to a waveguide is possible, but not necessary, and scanners that work for any properly polarized beam passing through the substrate can be made (Figure 12.9). ${ }^{19}$ For applications not requiring compatibility with waveguide devices, these bulk devices offer easier coupling, lower coupling and propagation losses, and improved beam quality. 
(a)

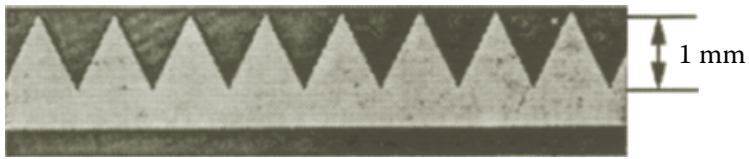

(b)

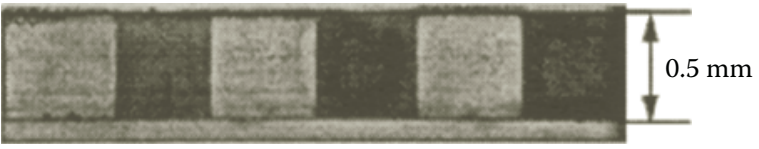

FIGURE 12.9

A bulk electro-optic wafer deflector using patterned domain inversion: (a) patterned Ta electrode used to define the geometry of the deflector; (b) etched Y cross-section of the sample showing domain inversion through the thickness of the wafer. (From Revelli, J.F. High-resolution electrooptic surface prism waveguide deflector: An analysis. Appl. Optics 1980, 19, 389-397. With permission.)

(a) Domain inverted region

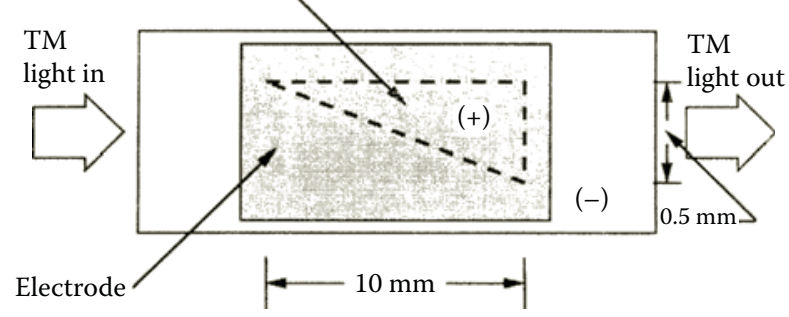

(b)

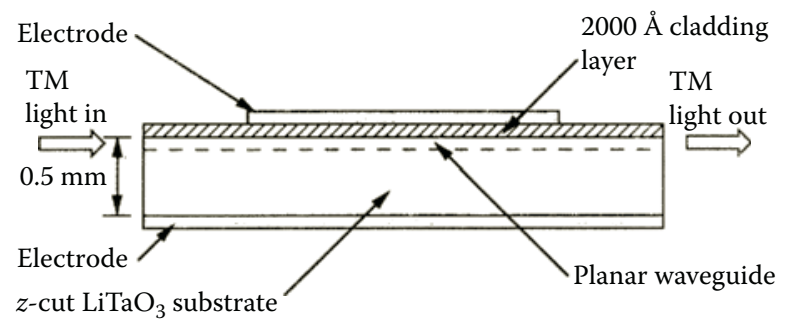

\section{FIGURE 12.10}

Geometry of the first electro-optic waveguide deflector using patterned domain inversion: (a) top view of the substrate showing the domain-inverted region; (b) cross section through the prism region. (From Lee, C.L.; Lee, J.F.; Huang, J.Y. Linear phase shift electrodes for the planar electrooptic prism deflector. Appl. Optics 1980, 19, 2902-2905. With permission.)

\subsubsection{Prismatic Poled Structures}

The first deflector of this type was a waveguide device fabricated in lithium tantalate, as shown in Figure 12.10. ${ }^{20}$ Domain inversion was achieved using patterned proton exchange followed by rapid thermal annealing. This process creates a domain-inverted region extending to a depth of 10-20 $\mu \mathrm{m}$. The planar waveguide was subsequently formed by proton exchange in $260^{\circ}$ pyrophosphoric acid. A $2000 \AA$ thick layer of $\mathrm{SiO}_{2}$ was deposited as a cladding layer before deposition and patterning of the final cover electrode, to reduce optical loss in the waveguide. Cylindrical lenses were used to edge-couple light into and out of the scanner.

Improved deflection sensitivity can be achieved by using thinner substrates, or by selectively thinning the substrate below the scanner using pulsed laser ablation. ${ }^{21}$ Selective 
thinning allows the internal field to be increased while maintaining mechanical strength around the border of the substrate.

\subsubsection{Rectangular Scanners}

The simplest scanner geometry is that for which the prisms are enclosed within a rectangular-shaped region. The general case is illustrated in Figure 12.11, where the active region is divided into an arbitrary number of variously shaped prisms. For $\theta_{\text {in }} \ll 1$ and $\Delta n \ll n$, the result of applying Snell's law at each interface is a cumulative deflection angle given by

$$
\mathrm{q}_{\mathrm{in}}=\sum_{i=1}^{N} \frac{\Delta n_{i}}{n} \cot f_{i}
$$

where $\Delta n_{i}$, is the total index change across the $i$ th interface, $N$ is the total number of interfaces in the scanner, and $\phi_{i}$ is the angle the $i$ th interface makes with the beam axis. Note that Equation 12.31 is valid regardless of the overall shape of the scanner, and each term in the sum is positive since both $\Delta n_{i}$ and $\phi_{i}$ change signs from one interface to the next. For rectangular scanners with a fixed $\left|\Delta n_{i}\right|=\Delta n$ at each interface, the scanning angle can be related to the width $W$ and total length $L$ of the device as follows:

$$
q_{\text {def }}=n q_{\text {in }}=\Delta n \sum_{i=1}^{N}\left|\cot f_{\mathrm{i}}\right|=\Delta n \sum_{i=1}^{N} \frac{l_{i}}{W}=\Delta n \frac{L}{W}
$$

Note that this is the same result as would be achieved for a scanner with a constant index gradient, from Equation 12.11. We therefore have the somewhat surprising result that the scanning angle does not depend on how many prisms are in the scanner, but only on the ratio $L / W$ ! To see why this is, note that as the number of interfaces increases, the angle of incidence becomes closer to normal thereby reducing the refraction at each interface. Consequently, the sum of the effects of all the interfaces is constant. The question of the effects of varying numbers of triangles is considered more closely below from a different point of view.

\subsection{Optimum Number of Triangles in Rectangular Scanners}

Considering the case of one interface, it is apparent that the scanning properties can, in fact, depend on the number of prisms or triangles. For a sufficiently large value of $L / W$, total

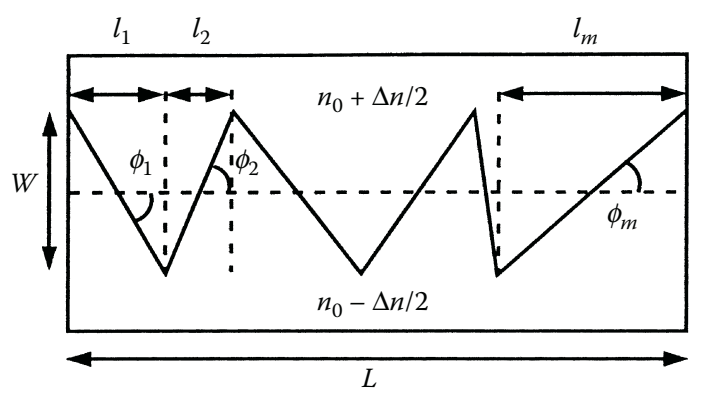

FIGURE 12.11

An arbitrarily divided rectangular prism scanner. (From Sasaki, H.; De La Rue, R.M. Electro-optic multichannel waveguide deflector. Electronics Letts. 1977, 13(10), 295-296. With permission.) 
internal reflection (TIR) can occur for one voltage polarity, but not the other. Consequently the scanning properties will be strongly asymmetric with respect to drive voltage polarity. On the other hand, if many interfaces are used, the incident angle at each interface will be sufficiently far from grazing that TIR cannot occur for practical values of drive voltage. Chen et al. ${ }^{20}$ have considered the scanning asymmetry as a function of the number of interfaces using ray-tracing simulations. As shown in Figure 12.12, the asymmetry in the scanning properties becomes negligible after about 10-15 interfaces.

Another consideration pertaining to the number of interfaces is the Fresnel transmission through the multiple interfaces. For small numbers of interfaces, the reflection at each interface can be high owing to the near grazing incidence (even TIR is possible, as mentioned above). This reflected light is diverted from the optical path, resulting in low transmission through the device. In the opposite limit of very many interfaces, the beam approaches normal incidence at each interface. Thus the reflection at each interface approaches a finite value, while the number of such reflections continues to increase. Thus the total reflection increases for both large and small numbers of interfaces. An optimum number of interfaces can be found that minimizes the total reflection, or equivalently, maximizes the transmission through the device. For $\Delta n / n \ll 1$ and $R \ll 1$, the normalized reflected intensity $R$ is approximately given by

$$
R=m\left[1+\left(\frac{L}{m W}\right)^{2}\right]^{2}\left(\frac{\Delta n}{2 n}\right)^{2}
$$

which has a minimum at the optimum number of interfaces:

$$
m_{\mathrm{opt}}=\sqrt{3} \frac{L}{W}
$$

Interestingly, this condition corresponds to filling the scanner with equilateral triangles. The behavior of the reflected intensity given by Equation 12.33 is illustrated in Figure 12.13.

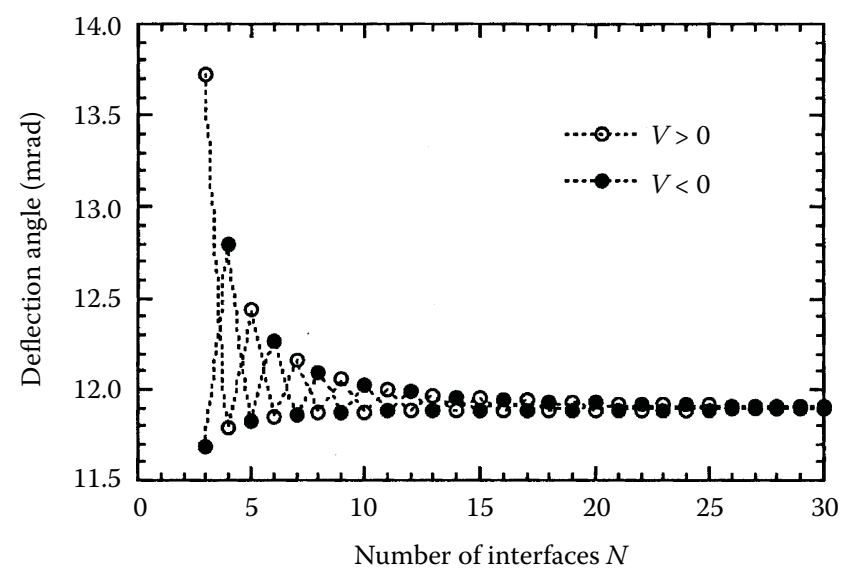

FIGURE 12.12

Symmetry of deflection angle versus the number of interfaces from ray tracing analysis. (From Takizawa, K. Electrooptic Fresnel lens-scanner with an array of channel waveguides. Appl. Optics 1983, 22(16), $2468-2473$. With permission.) 


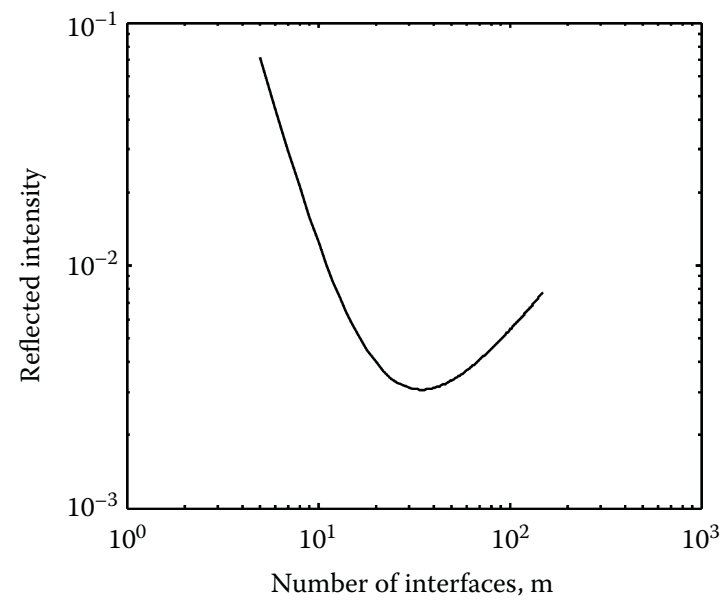

FIGURE 12.13

Normalized reflected intensity as a function of number of interfaces for $\Delta n / n=10^{-4}, L / W=20, m_{\mathrm{opt}}=35$.

Examination of this figure shows that although an optimum does exist, the reflected intensity is negligible over the practical range of interfaces large enough to satisfy the symmetry condition discussed above. We conclude that as long as the number of interfaces exceeds $10-15$, it is satisfactory to assume that the properties of the scanner are independent of the number of interfaces.

\subsection{Deflection Sensitivity for Rectangular Scanners}

To obtain the deflection sensitivity for a rectangular device, we need only to substitute the expression for $\Delta n$ into Equation 12.32. Using $E_{3}=V / h$ in Table 12.2 for crystals of symmetry $3 m$, the result is

$$
\frac{q_{\mathrm{def}}}{V}=\frac{n_{e}^{3} r_{33}}{h} \frac{L}{W}
$$

where $h$ is the thickness of the substrate, and the incident beam is polarized along the $z$-axis of the crystal (extraordinary wave). Bulk scanners will also work if the incident beam is polarized in the plane of a $z$-cut substrate (ordinary wave), but with reduced deflection. In this case, the deflection sensitivity is given by (Table 12.2):

$$
\frac{q_{\text {def }}}{V}=\frac{n_{0}^{3} r_{33}}{h} \frac{L}{W}
$$

It should be noted that Equations 12.35 and 12.36 are applicable to bulk devices; for waveguide devices, the voltage drop across the cladding layer must also be taken into account. This will result in a slight decrease in deflection sensitivity.

\subsection{Pivot Point Location for Rectangular Scanners}

The internal pivot point for rectangular scanners can be obtained directly from Equations 12.13 and 12.14:

$$
L_{\mathrm{P}, \text { in }}=\frac{L}{2}
$$


TABLE 12.2

Eigenvalues and Eigenvectors for $\mathrm{LiNbO}_{3}$ and $\mathrm{LiTaO}_{3}$ with an Electric Field Applied along the Optic Axis ${ }^{a}$

\begin{tabular}{|c|c|c|c|}
\hline Eigenvalue & Eigenvector & Index & $\Delta n$ \\
\hline$\frac{1}{n_{o}^{2}} \quad r_{13} E_{3}$ & $<100>$ & $n_{o}-\frac{\Delta n}{2}$ & $n_{o}^{3} r_{13} E_{3}$ \\
\hline$\frac{1}{n_{o}^{2}} \quad r_{23} E_{3}$ & $<010>$ & $n_{o}-\frac{\Delta n}{2}$ & $n_{o}^{3} r_{23} E_{3}$ \\
\hline$\frac{1}{n_{e}^{2}} \quad r_{33} E_{3}$ & $<001>$ & $n_{e}-\frac{\Delta n}{2}$ & $n_{e}^{3} r_{33} E_{3}$ \\
\hline
\end{tabular}

a $\Delta n$ is defined as in Table 12.1. Note that for these crystals $r_{13}=r_{23}$.

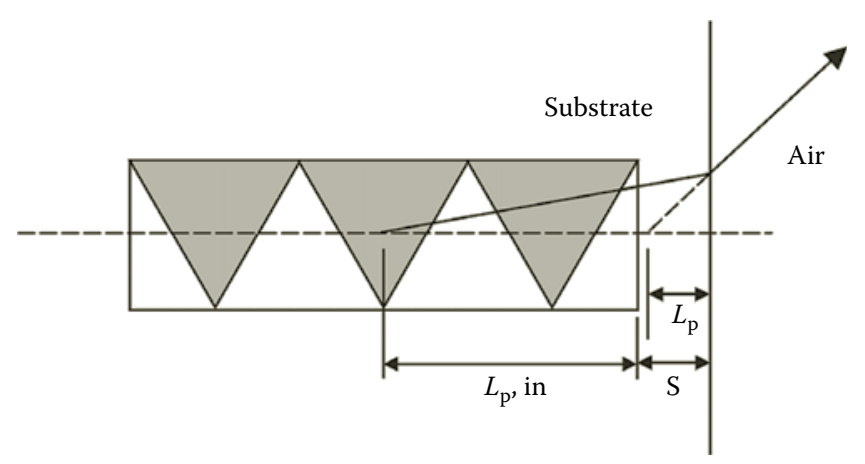

FIGURE 12.14

Shift of the apparent location of the pivot point by substrate refraction, including the effect of a spacing $s$ between the output of the scanner and the substrate edge.

In practical geometries, there is usually a spacing $s$ between the output plane of the scanner and the edge of the crystal, as shown in Figure 12.14. This spacing provides a longer electrical creepage path around the end of the scanner, and allows for cutting and polishing operations to be performed without impinging on the active scanner area. In this case, the location of the pivot point as viewed from outside of the crystal is given by (cf. Equation 12.15)

$$
L_{P}=\frac{1}{n}\left[L_{\mathrm{P}, \mathrm{in}}+s\right]
$$

\subsubsection{Trapezoidal Scanners}

One difficulty with the design of rectangular scanners results from the beam displacement within the scanner, as given by Equations 12.12 and 12.13. Clearly the width of the scanner must be increased to accommodate this displacement, but increasing the width reduces the deflection sensitivity. However, because of the shape of the trajectory, this increase is only needed at the output of the scanner. As discussed earlier, one way to address this is to focus the beam through the scanner so that the reduction in output beam diameter is comparable to the displacement. However, we saw that this technique reduces the number 
of resolvable spots. Another possibility is to increase the width of the output forming a trapezoidal shape (Figure 12.15).

\subsection{Deflection Sensitivity of Trapezoidal Scanners}

The deflection angle for a trapezoidal scanner is given by ${ }^{5}$

$$
q_{\text {in }}=\frac{\Delta n}{n} \frac{L}{W_{1}-W_{0}} \ln \left(\frac{W_{1}}{W_{0}}\right)
$$

Re-expressing this in terms of the external deflection angle and substituting for the change in refractive index gives the deflection sensitivity

$$
\frac{q_{\text {def }}}{V}=\frac{n_{e}^{3} r_{33}}{h} \frac{L}{W_{1}-W_{0}} \ln \left(\frac{W_{1}}{W_{0}}\right)
$$

\subsection{Pivot Point Location for Trapezoidal Scanners}

The displacement of the beam at the output of a trapezoidal scanner is given by ${ }^{5}$

$$
X(L)=\frac{\Delta n}{n} \frac{L}{W_{1}-W_{0}}\left[\frac{W_{1}}{W_{1}-W_{0}} \ln \left(\frac{W_{1}}{W_{0}}\right)-1\right] L
$$

The internal pivot point is obtained using the definition (14) along with Equations 12.39 and 12.41. The result is

$$
L_{P, \text { in }}=\left[\frac{W_{1}}{W_{1}-W_{0}}-\frac{1}{\ln \left(W_{1} / W_{0}\right)}\right] L
$$

The pivot point as viewed externally can be computed from Equation 12.38, as before.

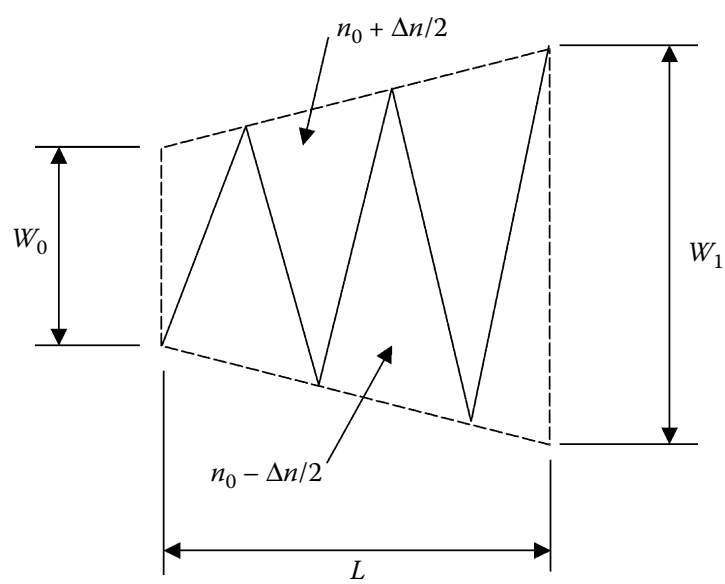

\section{FIGURE 12.15}

Geometry of a trapezoidal prism scanner. (From Chiu, Y.; Zou, J.; Stancil, D.D.; Schlesinger, T.E. Shape-optimized electrooptic beam scanners: Analysis, design, and simulation. J. Lightwave Technol. 1999, 17(1), 108-114. With permission.) 


\subsection{Comparison of Trapezoidal and Rectangular Scanners}

The increase in deflection sensitivity compared to rectangular scanners can be illustrated by considering rectangular and trapezoidal scanners with the same lengths, and the width of the rectangular scanner equal to the average of the input and output widths of the trapezoidal device. Thus, if $W_{R}$ is the width of the rectangular scanner and $W_{0}, W_{1}$ are the input and output widths of the trapezoidal scanner, we require

$$
W_{R}=\frac{W_{0}+W_{1}}{2}
$$

Assuming the same maximum index difference $\Delta n$ Figure 12.16 shows the improvement gained by the trapezoidal geometry. The improvement is modest $(<10 \%)$ for $W_{0} / W_{R}>0.5$. Since the usual case is that the beam diameter is much larger than the output displacement, $W_{0} / W_{1}$ and $W_{0} / W_{\mathrm{R}}$ are normally only slightly smaller than unity, yielding a typical improvement of a few percent.

\subsubsection{Horn-Shaped Scanners}

Since the scanning sensitivity is diminished as the width increases, the optimal solution is to increase the width gradually so as to track the beam trajectory. If the change in beam diameter through the scanner is neglected, the shape of the scanner can be obtained by simply adding a constant offset to the beam displacement, as shown in Figure 12.17a. The width of the scanner can therefore be written in the form

$$
W(z)=W_{0}+2 X_{\max }(z)
$$

where $X_{\max }(z)$ is the displacement at position $z$ with maximum voltage applied. The factor of 2 accommodates bipolar operation of the scanner. The general shape $W(z)$ has been obtained by Chiu et al., 5 and is plotted in terms of normalized coordinates in Figure 12.17b.

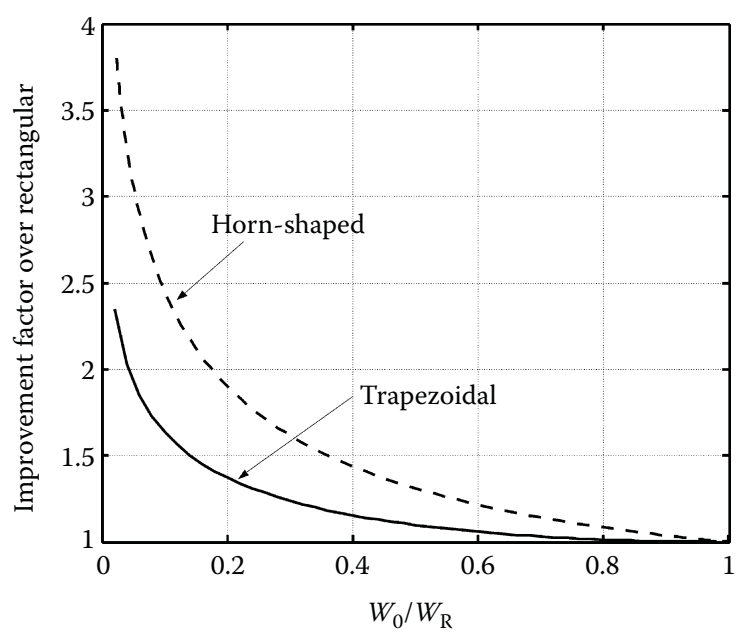

FIGURE 12.16

Comparison of scanning sensitivity of rectangular, trapezoidal, and horn-shaped scanners. (From Chiu, Y.; Zou, J.; Stancil, D.D.; Schlesinger, T.E. Shape-optimized electrooptic beam scanners: Analysis, design, and simulation. J. Lightwave Technol. 1999, 17(1), 108-114. With permission.) 
(a)

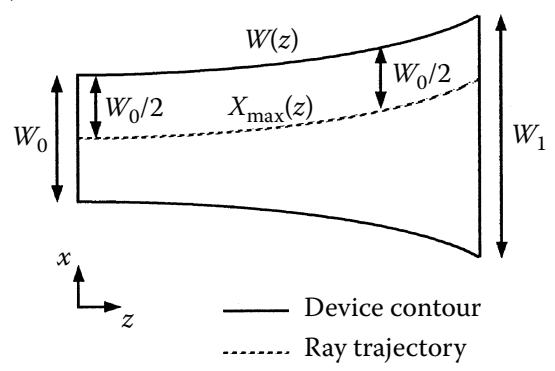

(b) 14

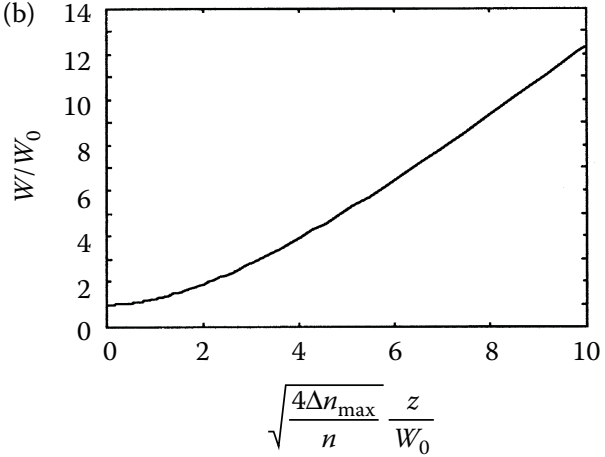

\section{FIGURE 12.17}

Normalized shape-optimized scanner design curve: (a) reference geometry and (b) normalized shape contour (from Chiu, Y.; Zou, J.; Stancil, D.D.; Schlesinger, T.E. Shape-optimized electrooptic beam scanners: Analysis, design, and simulation. J. Lightwave Technol. 1999, 17(1), 108-114. With permission.) Tabulated values are given in Table 12.3.

\section{TABLE 12.3}

Tabulated Values for the Normalized Horn-Shaped Scanner Curve Shown in Figure 12.17

\begin{tabular}{lrrrrrrr}
\hline$Z^{*}$ & $W^{*}$ & $Z^{*}$ & $W^{*}$ & $Z^{*}$ & $W^{*}$ & $Z^{*}$ & $W^{*}$ \\
\hline 0.00 & 1.00 & 2.50 & 2.49 & 5.00 & 5.36 & 7.50 & 8.84 \\
0.25 & 1.05 & 2.75 & 2.73 & 5.25 & 5.68 & 7.75 & 9.21 \\
0.50 & 1.11 & 3.00 & 2.99 & 5.50 & 6.01 & 8.00 & 9.58 \\
0.75 & 1.21 & 3.25 & 3.26 & 5.75 & 6.35 & 8.25 & 9.96 \\
1.00 & 1.34 & 3.50 & 3.53 & 6.00 & 6.69 & 8.50 & 10.34 \\
1.25 & 1.48 & 3.75 & 3.82 & 6.25 & 7.04 & 8.75 & 10.73 \\
1.50 & 1.65 & 4.00 & 4.11 & 6.50 & 7.39 & 9.00 & 11.11 \\
1.75 & 1.83 & 4.25 & 4.41 & 6.75 & 7.75 & 9.25 & 11.50 \\
2.00 & 2.04 & 4.50 & 4.72 & 7.00 & 8.11 & 9.50 & 11.89 \\
2.25 & 2.26 & 4.75 & 5.03 & 7.25 & 8.47 & 9.75 & 12.29 \\
& & & & & & 10.00 & 12.68 \\
\hline
\end{tabular}

$$
Z^{*}=\frac{z}{W_{0}} \sqrt{\frac{4 \Delta n_{\max }}{n}} W^{*}=W / W_{0} .
$$

a These values can be used for designing masks for scanner fabrication.

To facilitate use of this curve in designs, values of the normalized width as a function of $z$ are tabulated in Table 12.3.

The shape of the optimal curve allowing for the change in beam diameter through the scanner depends on the Gaussian beam parameters. Figure 12.18 shows a simulation of an optimal scanner with the beam focused on the output plane. ${ }^{22}$ The simulation was performed using the beam propagation method (BPM). ${ }^{23,24}$

\subsection{Deflection Sensitivity of Horn-Shaped Scanners}

The deflection angle as a function of $\Delta n$ for an optimum horn-shaped scanner is

$$
q_{\text {in }}=\frac{\Delta n}{n} \sqrt{\frac{n}{\Delta n_{\max }} \ln \left(\frac{W_{1}}{W_{0}}\right)}
$$




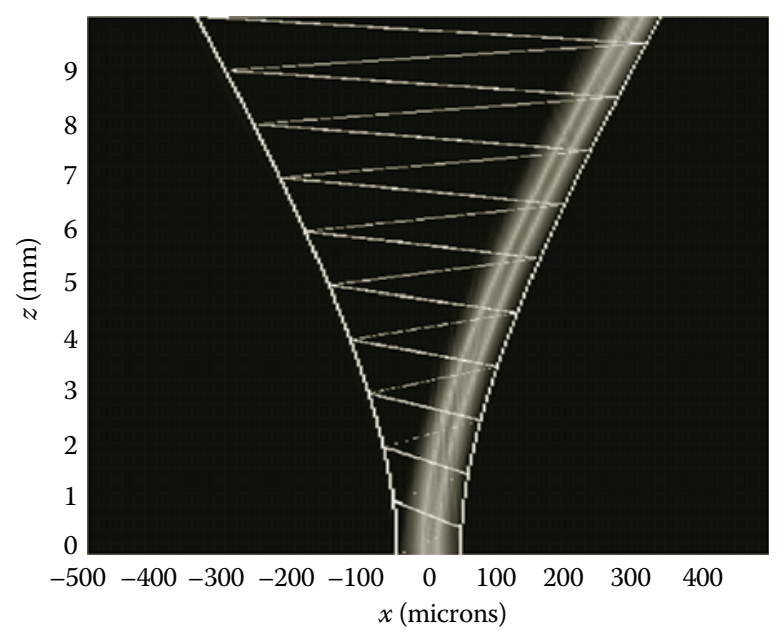

\section{FIGURE 12.18}

Simulation of horn-shaped scanner operation using the beam propagation method (BPM). Parameters are $W_{0}=92 \mu \mathrm{m}, W_{1}=678 \mu \mathrm{m}, L=10 \mathrm{~mm}, \lambda_{0}=0.6328 \mathrm{~mm}, n_{e}=2.1807$ (lithium tantalate), and $\Delta n=2.1 \times 10^{-3}$. The radius of the beam waist is $30 \mu \mathrm{m}$ and is focused at the output of the scanner. (From Fang, J.C.; Kawas, M.J.; Zou, J.; Gopalan, V.; Schlesinger, T.E.; Stancil, D.D. Shape-optimized electrooptic beam scanners: experiment. IEEE Photonics Technol. Lett. 1999, 11(1), 66-68. With permission.)

with a maximum value of

$$
q_{\mathrm{in}, \max }=\sqrt{\frac{\Delta n_{\max }}{n} \ln \left(\frac{W_{1}}{W_{0}}\right)}
$$

where $\Delta n_{\max }$ is the index variation across the scanner with the maximum applied voltage to be used. The deflection sensitivity is obtained by multiplying Equation 12.45 by $n$ and substituting for $\Delta n$ from Table 12.1. The result for crystals with symmetry $3 m$ (e.g., lithium niobate) is

$$
\frac{q_{\text {def }}}{V}=n_{e}^{2} \sqrt{\frac{r_{33}}{h V_{\max }} \ln \left(\frac{W_{1}}{W_{0}}\right)}
$$

\subsection{Pivot Point Location of Horn-Shaped Scanners}

The unipolar displacement at the output of the horn scanner is

$$
X(L)=\frac{\Delta n}{\Delta n_{\max }} \frac{W_{0}}{2}\left[\frac{W_{1}}{W_{0}}-1\right]
$$

Taking the ratio of Equations 12.48 and 12.45 gives the pivot point:

$$
L_{\mathrm{P}, \text { in }}=\frac{\left(W_{0} / 2\right)\left[\left(W_{1} / W_{0}\right)-1\right]}{\sqrt{\left(\Delta n_{\max } / n\right) \ln \left(W_{1} / W_{0}\right)}}
$$


Remarkably, the fact that $\Delta n$ drops out of this equation means that a well-defined pivot point exists (i.e., $L_{P, \text { in }}$ does not depend on voltage), even in such a complex horn-shaped device.

\subsection{Comparison of Horn-Shaped Scanners with Trapezoidal and Rectangular Scanners}

The deflection sensitivity of a horn-shaped scanner with the same input width, output width, and length as a trapezoidal scanner is shown in Figure 12.16, normalized to that of a rectangular scanner with the same average width. The improvement over trapezoidal and rectangular scanners is clear.

A summary of the design equations for rectangular, trapezoidal, and horn-shaped scanners is presented in Table 12.4 .

\subsubsection{Domain Inverted Total Internal Reflection Deflectors}

Domain inversion in ferroelectric crystals can also be used to create relatively long, straight interfaces within a bulk crystal. Since the domains are antiparallel across the interface, an applied electric field will result in a step change in the index of refraction. If a light beam intersects this interface at very high angles (i.e., near grazing incidence) a state of TIR can result (Figure 12.19).

Eason and coworkers report an analysis of such a device, ${ }^{25}$ with an application to telecommunications switching in mind. Such a device would typically be operated in a digital fashion, ON or OFF, in order to steer a collimated beam into one or another optical fiber (with appropriate collection optics on the end of each fiber). Compared to a scanner composed of many triangles, such a TIR device will exhibit greater deflection for the same voltage and device size. This gives some advantage by shortening the package length since the two beams separate faster providing more room for the output fibers and their collimation optics. A further advantage is that TIR device performance may be engineered to exhibit nearly polarization independent performance, while triangular prisms are strongly polarization dependent.

\subsubsection{Domain Inverted Grating Structures}

The component based upon patterned domains that has received the most attention in recent years is the quasi-phase-matched grating for second harmonic generation (SHG). ${ }^{13-17}$ The frequency shifting effect occurs in a "periodically poled" region in the crystal, which is produced by forming many precisely spaced parallel domains in materials such as lithium niobate or KTP. ${ }^{17}$ For instance, some early efforts were focused on producing blue light from IR laser diodes for use in data storage applications.

In SHG applications, the light propagates perpendicular to the poled stripes, and generally there is no applied electric field. By rotating the incident beam to near grazing incidence and allowing for the application of an electric field, an EO Bragg grating is produced. This is analogous to an AO Bragg deflector where the electric field rather than the acoustic intensity controls the distribution of the light into the various orders.

Gnewuch and coworkers have designed and tested such a device operating at $633 \mathrm{~nm} .{ }^{26}$ Similar to the TIR device above, the incident light can be switched to two different positions. The advantage of this structure, however, is that it operates at approximately 25 $\mathrm{V}$, versus $500 \mathrm{~V}$ for a prismatic domain poled device operating with a similarly sized optical beam. This offers the potential for enormous electrical power savings during 

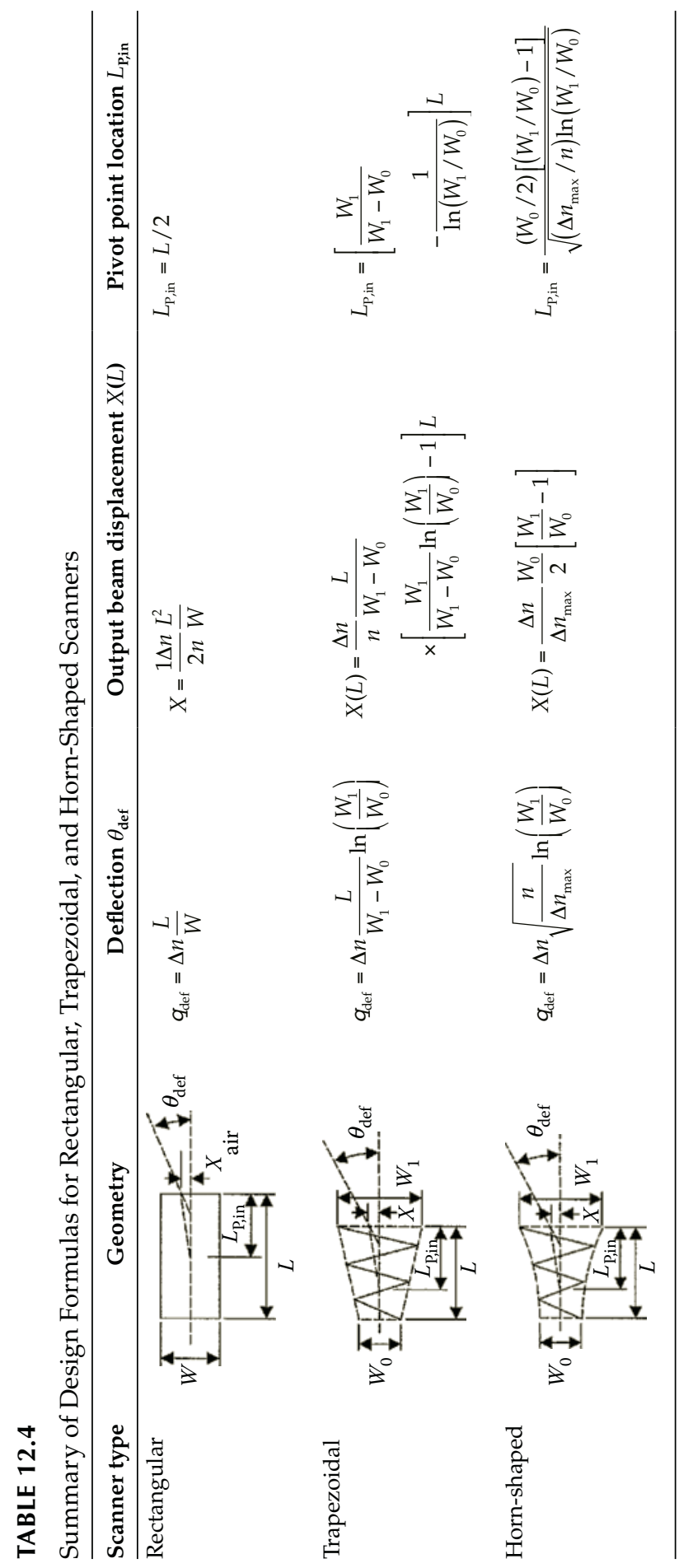

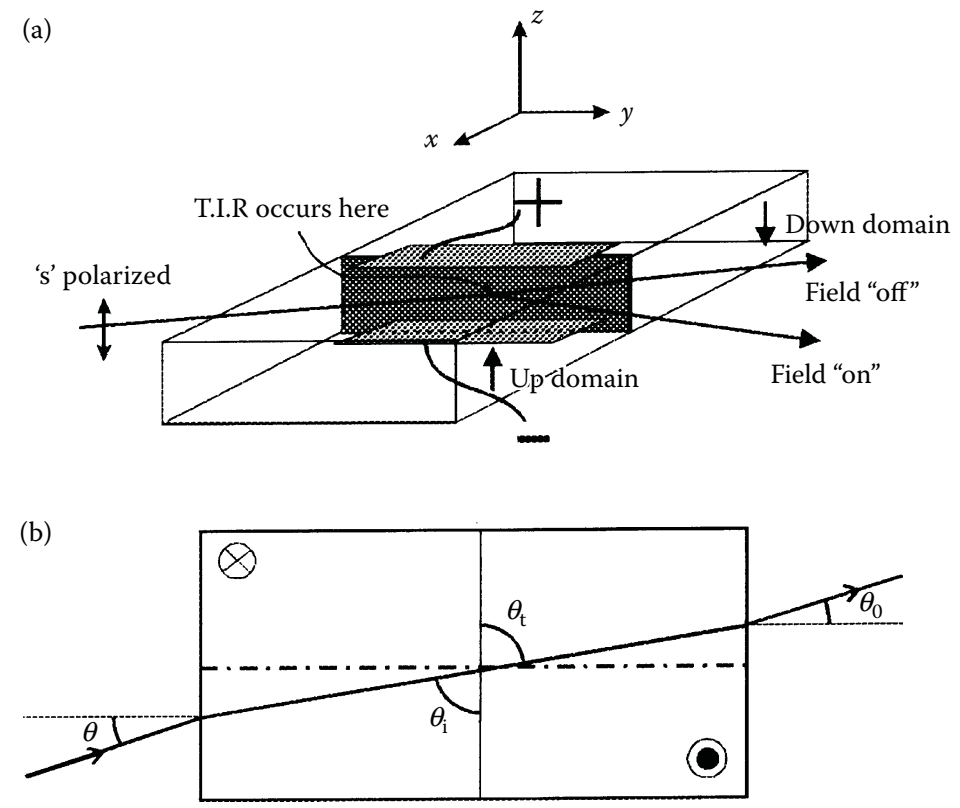

FIGURE 12.19

(a) Schematic of domain engineered total internal reflection (TIR) deflector; (b) plan view of scanner showing grazing angle of the input optical beam to the poled interface. (From Eason, R.; Boyland, A.; Mailis, S.; Smith, P.G.R. Electro-optically controlled beam deflection for grazing incidence geometry on a domain-engineered interface in $\mathrm{LiNbO}_{3}$. Optics Commun. 2001, 197, 201-207. With permission.)

high-frequency operation. Without acoustic velocity issues, the EO version of the Bragg deflector can operate at high speeds and handle higher beam powers than an $\mathrm{AO}$ version could.

\subsubsection{Other Poled Structures}

The power of patterned ferroelectric domain inversion is that a wide variety of optical components can be fabricated by designing masks with appropriate shapes. For example, although patterning the optimal horn-shape photolithographically is straightforward, the fabrication of a horn-shaped scanner by assembling discrete prisms would be prohibitively difficult. Further, with patterned domain inversion multiple components can be easily integrated on a common substrate.

As another example, EO cylindrical lenses can be formed by patterning oval, semicircular, or circular inverted domains. ${ }^{27,28}$ Stacks of such lenses are readily combined with a prism scanner. ${ }^{29}$ An optical integrated system of this type could be used, for example, to collimate the light from a fiber before entering the scanner. It should be noted, however, that lenses made in this way are cylindrical lenses, and hence only focus in the plane of the substrate. This is of no consequence in planar waveguide devices, but must be considered if bulk operation is desired.

A periodically poled structure can also be integrated with prism scanners to realize a device capable of generating and steering blue light. ${ }^{30}$ The SHG conversion efficiency of this bulk integrated device was relatively low, however, since the optical intensity in 

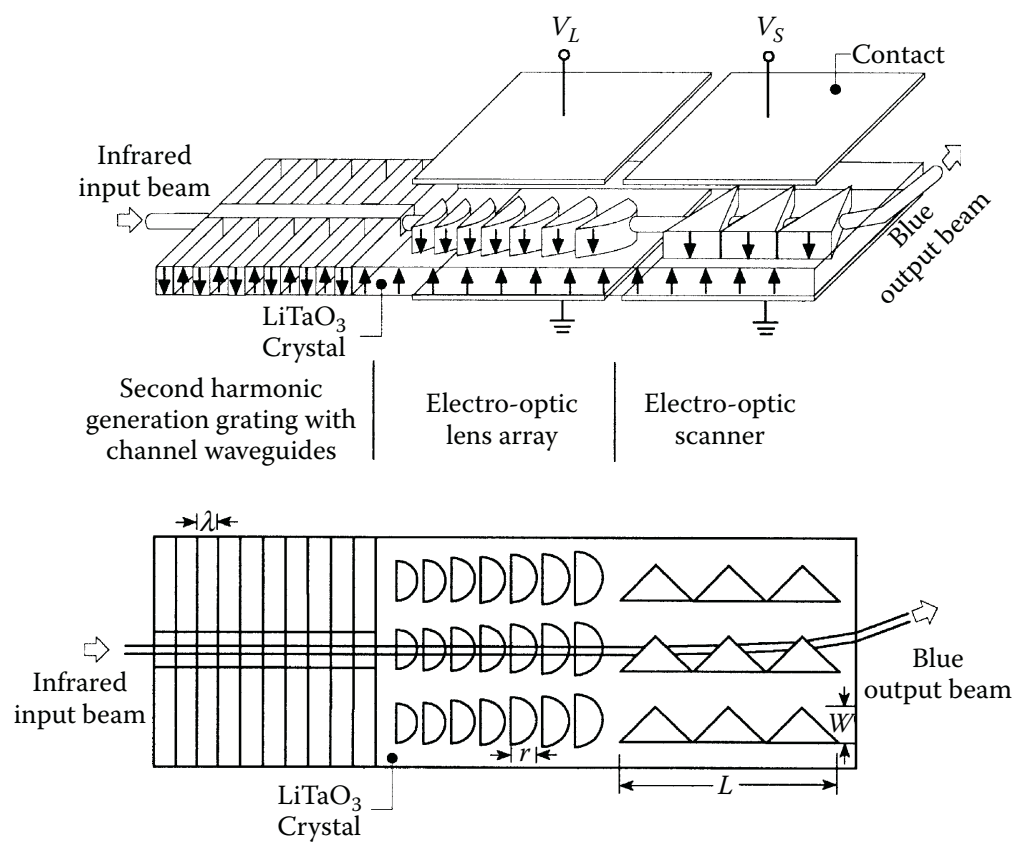

FIGURE 12.20

Schematic of an integrated device containing a channel waveguide with an SHG grating, collimating lens stack, and scanner. (From Chiu, Y.; Gopalan, V.; Kawas, M.J.; Schlesinger, T.E.; Stancil, D.D.; Risk, W.P. Integrated optical device with second-harmonic generator, electrooptic lens, and electrooptic scanner in $\mathrm{LiTaO}_{3}$. J. Lightwave Technol. 1999, 17(3), 462-465. With permission.)

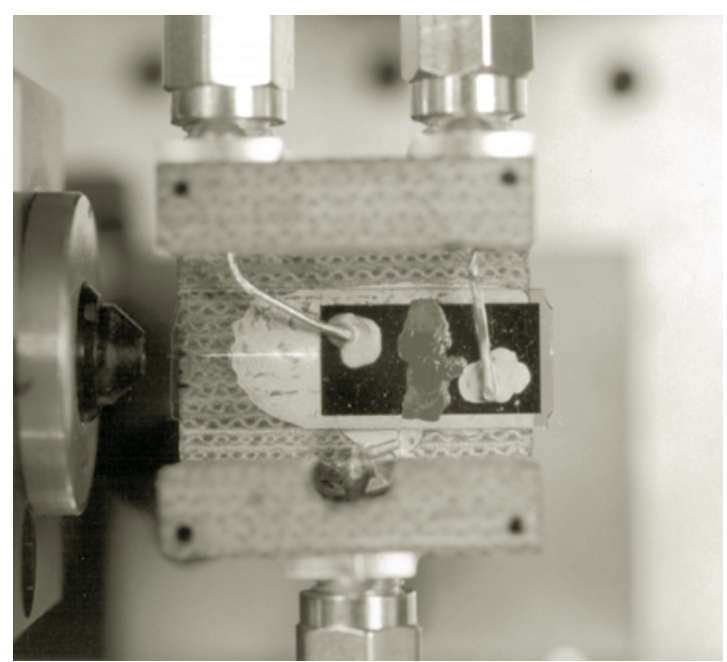

FIGURE 12.21

Photograph of the device described in Figure 12.20. Blue light generated by the SHG waveguide can be seen on the left, followed by the lens and scanner electrodes. 
the grating was limited by the focused beam diameter. To achieve the highest conversion efficiency, the light must be confined to a channel waveguide.

The issue of low conversion efficiency encountered with the first SHG scanner device can be addressed by combining the above elements to form an integrated SHG grating, lens stack, and scanner, as shown in Figures 12.20 and $12.21 .{ }^{31}$ The channel waveguide in the grating keeps the optical intensity high to maximize the nonlinear frequency doubling efficiency. The output of the channel waveguide then opens into a planar waveguide, and the beam subsequently diverges. A lens stack is next used to collimate the beam before entering the scanner.

\subsection{ELECTRONIC DRIVERS FOR ELECTRO-OPTIC DEFLECTORS}

\subsubsection{Overview}

A primary consideration when applying EO deflector devices as part of a system, and an issue often raised by potential users, is the electronic driver. The driver is essentially an amplifier, converting a low-voltage control signal to the higher voltage required to achieve the desired optical index change. It is usually custom-designed for each application, requires specialized circuit design skills and may present safety hazards. Depending on the specific system requirements, the design challenges can vary; however, the highvoltage (HV) nature of these devices can be the single most restricting factor, as component availability can restrict the design space. Also, the HV supplies required to power the driver(s) can be a considerable cost and design challenge.

Other parameters that can influence the design are: switching speeds; repetition rate; switch/component package density requirements; electromagnetic interference (EMI) and radio frequency interference (RFI) considerations; switching power efficiency; and thermal considerations.

Significant strides in recent years in power field effect transistor (FET) and insulated gate dipolar transistor (IGBT) technology have alleviated many practical constraints and enabled power densities and performance that were not possible in earlier years. These devices, developed by Motorola, IXYS, International Rectifier, Toshiba, ST Microelectronics, and others, primarily for the motor drive/control industry, can be well suited for EO scanner drive applications. Also, very fast HV diodes by these same manufacturers and surface mount HV capacitors from Johansson Dielectric and others have driven achievable performance up considerably.

\subsubsection{High-Voltage Power Supplies}

Most EO scanner drivers rely on a constant high voltage supply as a subsystem. For instance, a modulator system can be realized by switching one electrode of an EO element between a high voltage and ground while the other electrode is held at ground. Benchtop HV power supplies are readily available from several manufacturers including Spellman, Ultravolt, Trek, and others. The market for application-specific HV supplies is not large and, as such, cost can often be quite high and standard packaging options somewhat limited. In many cases it is desirable to design a custom HV supply that suits the application.

There are several design topologies available for HV power supply design. Whether the input power is AC line voltage or a DC supply, a voltage boost converter of some type 
needs to be employed. A typical boost converter is discussed and then some higher efficient topologies covered.

\subsubsection{Conventional Boost Converters}

Switching supply topologies, such as boost converters, can be utilized when a high voltage needs to be generated from a much lower one. Typical boost converters consist of a switching transistor (Q1), usually a FET, with an inductor (L1) connected between the drain and the low voltage supply. Current is transferred discontinuously at the switching frequency, and the stored energy amount and pulse duration are proportional to the output voltage feedback signal (Figure 12.22).

The load in a boost converter is usually fed through a rectifying diode. The current in the inductor $I_{L 1}(\mathrm{pk})=\left(V_{\mathrm{dc}}{ }^{*} t_{\mathrm{on}}\right) / L 1$, ramps up linearly during the ON cycle of the FET. The energy stored is $E=1 /\left(2 * L 1 * I_{L 1}^{2}(p k)\right)$. When the FET is turned off that energy is then transferred to the load via the rectifying diode. A portion of $V_{\text {out }}$ is fed back through a pulse width modulation (PWM) converter to control the desired pulse width of the FET drive. Boost converters are typically only used in lower power applications of less than $10 \mathrm{~W}$.

\subsubsection{Flyback Converters}

In many HV applications with moderate to high power requirements, the size of the inductor in the boost converter needed to store the proper amount of energy becomes unwieldy, and losses become high. In these cases, a transformer can replace the boost converter inductor. This topology is referred to as a flyback converter. A schematic example is shown in Figure 12.23.

The basic operation of a flyback converter is that when the FET is turned ON, current ramps up at a rate $\mathrm{d} i / \mathrm{d} t=\left(V_{\mathrm{dc}}\right) / L_{\mathrm{pm}}$, where $L_{\mathrm{pm}}$ is the magnetizing inductance of the primary winding of transformer T1. When the FET is subsequently turned off the current has

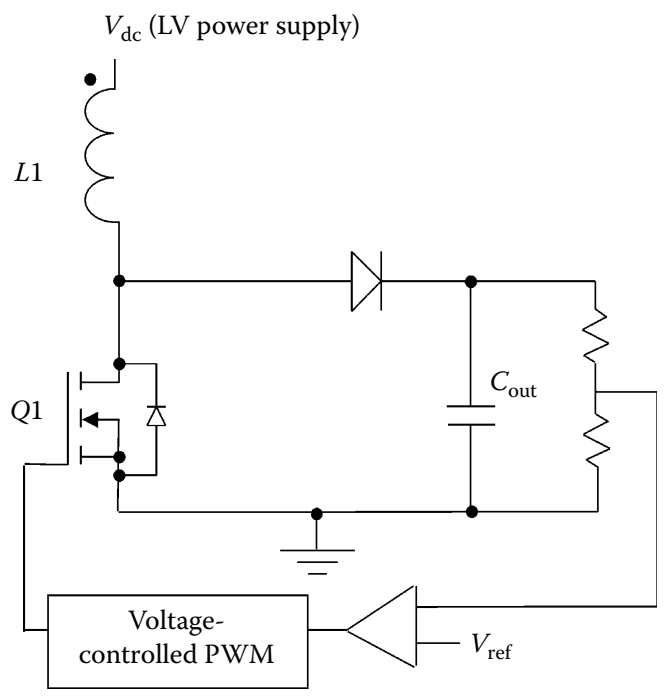

FIGURE 12.22

Schematic of conventional boost converter. The driver circuit for the EO element is connected across the output storage capacitor, $C_{\text {out }}$. This type of high voltage supply is typically used in applications requiring less than 10 $\mathrm{W}$ of output power. 


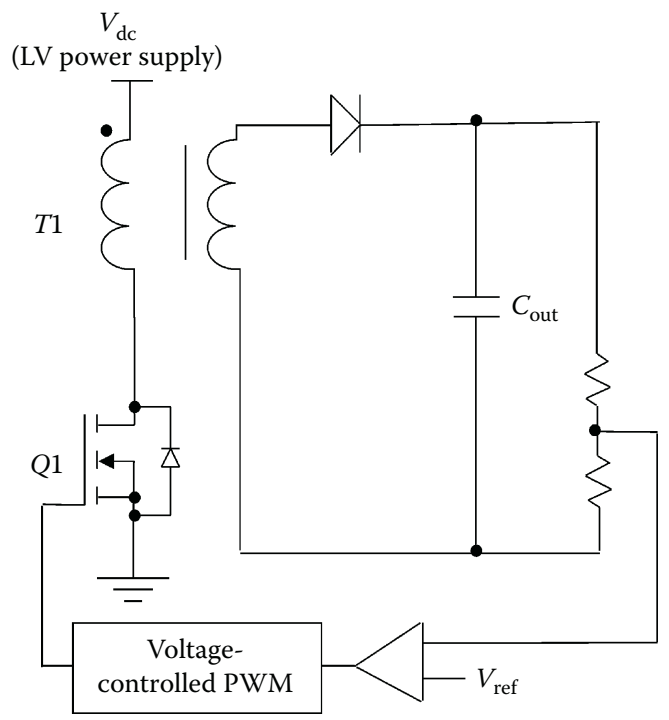

FIGURE 12.23

Schematic of a flyback converter.

ramped to the $I_{\mathrm{pk}}=\left(V_{\mathrm{dc}}\right)^{*} T_{\mathrm{on}} / L_{\mathrm{pm}}$ thus storing the energy $E=L_{\mathrm{pm}}{ }^{*}\left(I_{\mathrm{pk}}\right)^{2} 2$. With the FET turned OFF, the magnetizing inductance causes an instantaneous reversal in polarities of all windings' voltages and the primary current transfers to the secondary as $I_{\mathrm{s}}=I_{\mathrm{pk}}\left(N_{\mathrm{p}} / N_{\mathrm{m}}\right)$ where $N_{\mathrm{p}}$ and $N_{\mathrm{m}}$ are the primary and secondary winding count. Using a higher voltage primary $V_{\mathrm{dc}}$ can help minimize transformer size and keep the $I_{\mathrm{pk}}$ to manageable levels. Also, one can employ multiple winding outputs to increase output voltage as needed or to select one of multiple output levels.

The flyback converter topology can be used for 5-150 W supplies for $V_{\text {out }} \leq 5000 \mathrm{~V}$. A limiting factor can be that the required current in the primary of the flyback transformer is not excessive while keeping within an acceptable, and realistic, transformer size. Efficiencies of the order of $85 \%$ and above are achievable.

\subsubsection{Digital Drivers}

Many EO systems such as telecommunications switches and display system optical beam modulators, can be realized using drivers with only two output states. The term "digital driver" is used here, although the voltages being considered can range to $1000 \mathrm{~V}$ and above, requiring far different components than digital logic circuits.

\subsubsection{Simple Totem Pole Circuits}

The simplest and highest speed driver for controlling a capacitive EO device is a "totem pole" or "half bridge" arrangement of FETs where the totem pole is connected across a HV power supply and the device is connected between the FETs to ground (Figure 12.24). Each FET is turned ON or OFF in turn to set the voltage across the output (load) EO device to either the high or low state. Both FETs are never turned $\mathrm{ON}$ at the same time-this would lead to a high current directly from the high voltage supply and would likely destroy the FETs unless proper limiting circuitry is used. 


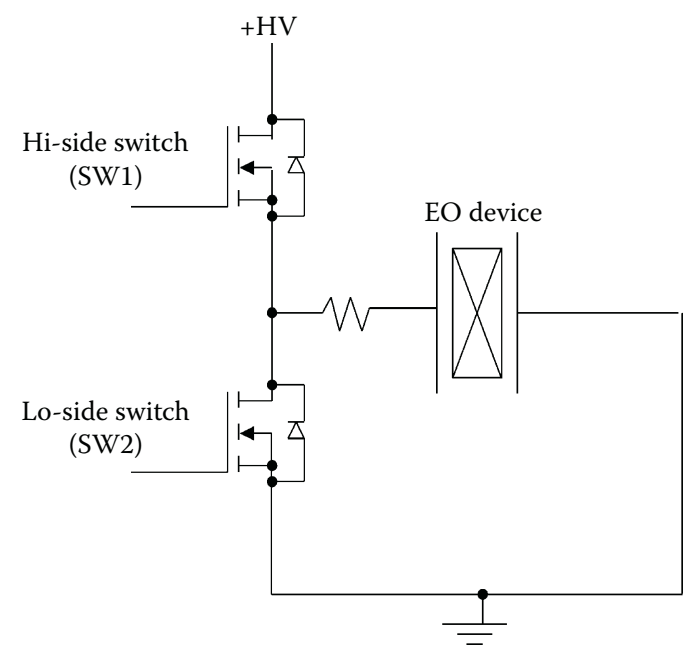

FIGURE 12.24

Schematic of a totem pole driver. The $+\mathrm{HV}$ lead of this driver would be attached to the high side of $C_{\text {out }}$ of a high-voltage power supply.

In some cases, the FETs would have resistors in series with them to dissipate some of the charge/discharge energy (without the resistors, nearly all of the energy is dissipated in the FETs during the switching transition, which can cause substantial heating). This will, of course, slow down the charging and discharging edges slightly. This straightforward totem pole results in a very high speed, although very lossy, method to drive the output capacitor, in this case, an EO scanner.

The high-side drive can be easily crafted from a p-channel FET where HV $<200 \mathrm{~V}$. However, many practical applications can require a higher voltage, and such FETs are not available. As such, n-channel FETs are recommended for most applications and can be utilized with a floating high-side gate drive. A simple implementation of this would be a transformer-coupled FET gate drive circuit.

It should be noted that this high-side gate driver must be crafted such that in the "dwell ON state," the gate drive must be able to keep the FET ON for the longest system dwell time, which may be an undetermined duration. If continuous (infinite) dwell is required then a refresh circuit should be added to the transformer-coupled FET gate driver circuit.

Gate drivers for the FETs can be crafted from discrete elements-many of those that were designed for the motor drive industry can be utilized. The main FETs must be selected for low junction capacitances rather than minimal $R_{\mathrm{ds}}($ on) as a primary constraint as these capacitances must be charged and discharged during the switching cycle and can be comparable to the load capacitance of the EO device itself and contribute significantly to circuit losses.

Devices such as the highly integrated totem pole driver ICs from International Rectifier (IR2213), ST Microelectronics (L6285), and others can be utilized for applications up to 1000 V. Beyond that, IGBTs can be employed. These devices have $T_{\text {on }} / T_{\text {off }}$ propagation delays and these parameters can drift significantly with temperature such that timing control circuitry must take it into account. Switching speeds on the order of $100 \mathrm{~V} / \mathrm{ns}$ are possible.

The maximum voltage rating of most devices in the circuit can be cut in half, thus greatly increasing the component choices and safety factors, by utilizing a full bridge drive where two totem poles are set up to switch $1 / 2 \mathrm{HV}$ each to the load. Efficiency benefits can also be realized as the loss from the two charge cycles can be less than one-half that of switching the 
entire HV in one step. Further, this topology enables "adiabatic" switching to be utilized such that further efficiency gains can be realized-this concept is covered in Section 12.4.3.2.

\subsubsection{Adiabatic Drivers}

Inherent in traditional switching logic design is the $C V^{2} / 2$ of energy that is dissipated every time a transistor is turned on to charge or discharge a capacitive load. This dissipation is a direct consequence of the fact that, in traditional switching logic configurations, charge for the load is taken from a power or ground rail and that the device to be charged initially sits at a fixed potential very different from that of the rail.

In a simple totem pole application of charging and discharging a load $\mathrm{C}$ to a voltage $V$, the energy dissipated to flip the output is $E=\frac{1}{2} C V^{2}$. This energy does not depend on the needed time to switch, nor the clock rate, but is strictly related to the energy transfer process. In fact, during the rising transition, the power supply delivers all the charge $Q=C V$ at voltage $V$, while during the falling transition that charge is returned at zero voltage. So, actually, the energy $E=C V^{2}$ is drawn from the HV supply, with half of the energy being stored in the load capacitor, and the other half being dissipated in losses.

Put another way, half of this energy is dissipated by the FET in the pull-up network (rising transition) and the other half by the pull-down network (falling transition), independent of how fast the transitions are. To reduce the dissipated energy, only methods that reduce the load capacitances or the supply voltage can be applied, but in any case they are strictly limited by the load $C$ and voltage $V$.

The term "adiabatic process" is most often applied to the thermodynamic cycle by which a gas, such as air, is heated or cooled by expansion or compression without an external source or sink of heat.

The energy transfer processes in the electronic driver can be done adiabatically if, during the rising (or falling) transition, the power supply delivers (or recovers) the charge to (or from) the load at a potential close to that of the source/supply potential. In other words, in order to implement adiabatic processes in a switching circuit, the switching devices should be turned on only when the source-drain voltage is zero, and source-drain voltage should be changed only while the device is off; and, if possible, given the desired performance of the circuit, any voltage change must be done as gradually as possible.

There are difficulties in implementing this solution, however. First, the logic must be designed so that switching transitions can occur only at suitable times (that is, only when there is no potential drop across the switching devices). Choreographing this timing can add considerable complexity as there are switching delays inherent in the FETs and gate driver elements. FET drivers and control circuitry also have temperature-dependent characteristics. Secondly, zero energy dissipation only occurs with arbitrarily slow switching: with realistic switching rates, the energy savings might not be enough to make up for the additional complexity. Lastly, adiabatic design relies on the assumption that one can efficiently provide the moving supply (in fact a clock) to the circuit that it drives. This last characteristic is not achievable with HV applications and is more suited for logic level designs.

In Figure 12.25, note that the two half bridges are configured such that the "lo-side" -HV switch is referenced to the -HV rail. This is fairly easily implemented as will be discussed later.

The full bridge circuit operates adiabatically as follows. Start with the crystal discharged, that is PH and NL are off (or open) while PL and NH are turned on. To charge the crystal, one of the grounded FETs, PL is turned OFF-this is adiabatic, in that it is 


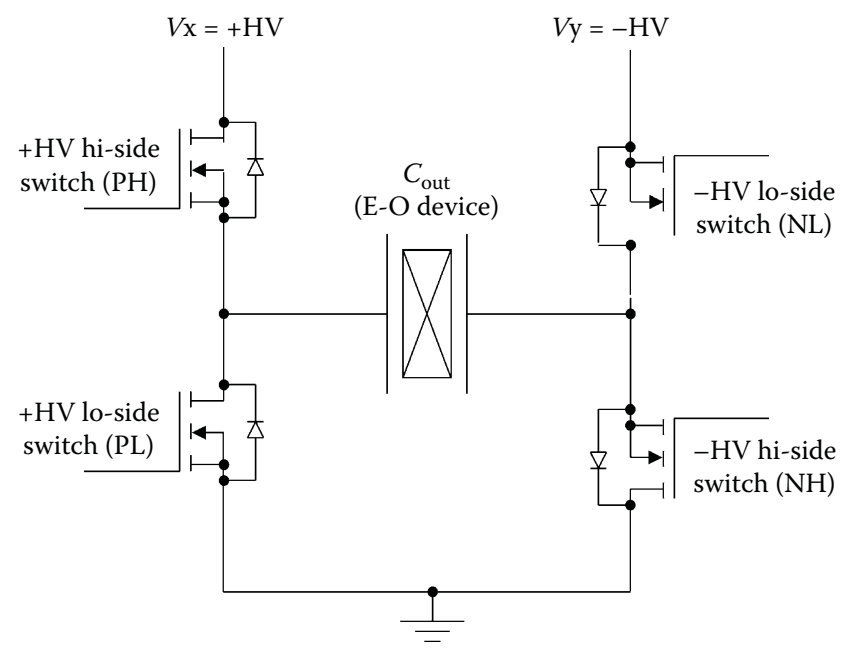

FIGURE 12.25

A dual totem pole driver. By using both a positive and a negative high-voltage supply, the absolute voltage level may be halved compared to a simple totem pole circuit. This can simplify some design tasks. This type of circuit can also be controlled to operate "adiabatically," which offers significant power savings.

done while no current is flowing $\left(V_{\mathrm{ds}} \sim 0 \mathrm{~V}\right)$. Only a very short period of time is required for it to fully turn off, then immediately turn ON switch PH. Obviously PL must be fully OFF before PH can begin to turn ON to avoid a catastrophic shoot-through condition HV to GND. Note the basic totem pole circuit always switched with $V_{\mathrm{ds}}$ at $\sim \mathrm{HV}$, leading to losses in the FET.

This switch transition will result in the following: the $+\mathrm{HV}$ device, $\mathrm{PH}$, will need to supply energy to charge the load and the parasitic capacitance of PL, $C_{\mathrm{PL}}$, which includes the capacitance of the protection diode, which is usually a significant component of the total load. Thus the energy required from the $+\mathrm{HV}$ supply is $\left(C_{\text {load }}+C_{\mathrm{PL}}\right)(\mathrm{HV})^{2}$. Since each $\mathrm{HV}$ supply is one-half the voltage of what it was in the simple totem pole arrangement, this translates to $\left(C_{\text {load }}+C_{\mathrm{PL}}\right) \mathrm{HV}^{2} / 4$ of energy drawn so far.

The next step, to fully charge the crystal, is to turn NH OFF (again while $V_{\mathrm{ds}} \sim 0 \mathrm{~V}$ ) and turn on NL. As before the -HV supply needs to charge up $C_{\text {load }}+C_{\mathrm{NH}}$. The required energy thus to turn $\mathrm{ON}$ the $C_{\text {load }}$ is:

$$
E=\left(C_{\text {load }}\right)(+H V)^{2}+\left(C_{\text {load }}\right)(-H V)^{2}
$$

Since each $\mathrm{HV}$ in this case is one-half the voltage compared to the simple totem pole case, the energy is $\left(C_{\text {load }}\right)(\mathrm{HV} / 4)+\left(C_{\text {load }}\right)(\mathrm{HV} / 4)$ or $\left(C_{\text {load }}\right)\left(\mathrm{HV}^{2} / 2\right)$, a saving of approximately half of the energy (minus that to charge the stray and parasitic capacitances of the additional switching device) for each cycle.

For the adiabatic discharge, NL is turned OFF, then NH is turned ON. This will force the recovery of half the charge in the crystal to go back to $+\mathrm{HV}$ supply (or $\mathrm{C}_{\text {load }}{ }^{*} \mathrm{HV}^{2} / 4$ of energy is recovered). Also, the parasitic capacitance of $C p(\mathrm{NL})$ will be dissipated and $C p(\mathrm{NH})$ will be charged to $V y$, requiring $C p(\mathrm{NH})^{*} V y^{*} 2$ energy from $V y$ supply. This total energy is equivalent to $C p(\mathrm{NH}) V y^{*} 2 / 4$. Finally, to completely discharge the crystal, we need to turn OFF PH and turn ON switch PL. 


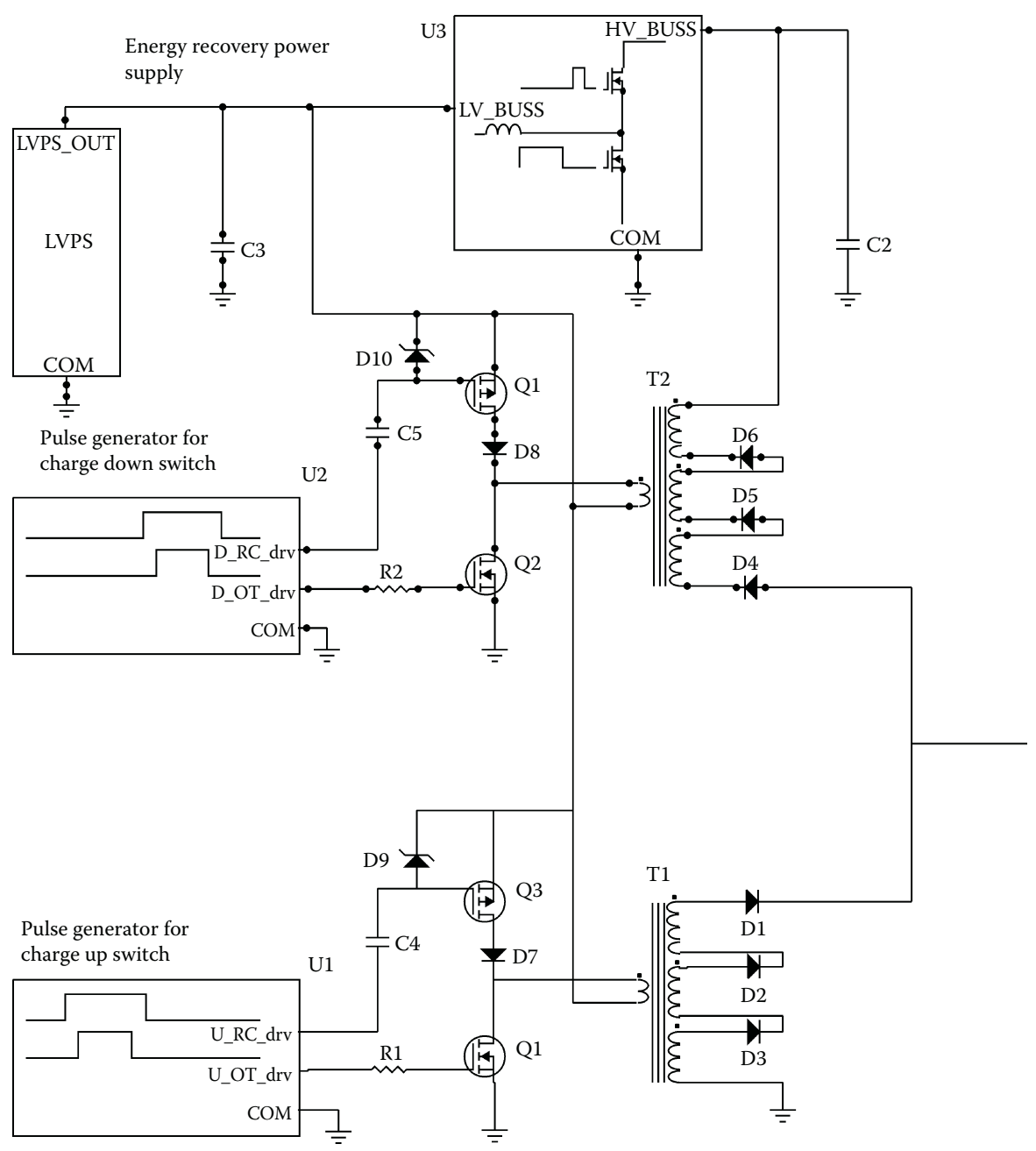

FIGURE 12.26

A schematic of flyback high-voltage supply with an energy recovery circuit, allowing for connection to an adiabatic driver circuit.

Of course, the HV supplies used in an adiabatic driver control scheme must be able to source and sink current-which is not true of most HV supplies. However, additional circuit elements can be added to the flyback converter to accommodate this requirement. Further gains in overall energy efficiency can be realized by making the HV power sink operate as an energy recovery system. One option for this is shown in Figure 12.26, where the energy pushed back to the HV rail is fed into a step-down DC-DC converter with the output connected to a system level low voltage bus. ${ }^{32}$

\subsubsection{Analog Drivers}

The wide variety of EO applications and elements leads naturally to a wide variety of drivers, especially for "nondigital" applications. Possible designs range from those using densely packaged hybrid circuitry to those using $4000 \mathrm{~V}$, or higher, vacuum tubes for 
control elements. A variety of IC op-amps are also available up to $600 \mathrm{~V}$, allowing for essentially arbitrary waveforms within the bandwidth of the amplifier.

Analog drivers are generally specified by their operating voltage and bandwidth. The bandwidth of a driver is always specified in relation to the load; the usable bandwidth of the driver will decrease as the load capacitance increases. For this reason, the cabling or other connection between the driver and the EO element must be considered in addition to the actual EO element load when specifying the driver, since they can sometimes dominate the overall system performance. Efficiency, single-ended or differential input, linearity, and other factors may also be specified.

A sampling of the variety of designs possible is:

1. Single ended: this is the simplest, most straightforward variety of driver. The driver is connected to one electrode on the EO device, with the other usually held at ground. Op-amps are typical single-ended drivers.

2. Differential: some amplifiers work best in the middle of their output voltage range. A differential driver is constructed using two amplifiers, each connected to one side of the EO element and each biased to one-half the peak voltage. As the voltage on one is raised, the other is lowered. Differential topologies can be used where deflection in either direction from nominal is required. They also effectively act as a single-ended design but with twice the voltage-which may be useful if FETs at the desired voltage are not available.

3. Resonant: since EO elements act as capacitors, they can be coupled with an inductor and driven at the resonant frequency of the pair. Voltage amplification is possible, and standard techniques can be used to synchronize the circuit with other elements of the complete optical system.

4. Transformer coupled: voltage amplification is also possible using a transformer. This type of circuit may be operated in a resonant mode, or to produce some other waveform. Transformer coupling is best suited to periodic waveforms since the bandwidth of a transformer can be limited, and special tuning and compensation techniques may be required for complex waveforms. Trianglewave drive voltages, useful for some display applications, can be done very effectively.

\subsection{PROPERTIES AND SELECTION OF ELECTRO-OPTIC MATERIALS}

\subsubsection{General}

The optical performance of an EO deflection system depends on the material chosen, the operating electric field, and the characteristics of the beam to be deflected (wavelength, diameter, divergence, $M^{2}$ quality factor, and so on). Unfortunately for system designers, there is no one "best" material that meets most application requirements.

Most practical EO materials are crystals with anisotropic properties, and almost all of these are grown by crystal suppliers because they are not abundant in nature. Properties can vary by manufacturer and grade, making it important to work in concert with the material suppliers when selecting a material; they are also good sources of material prop- 
TABLE 12.5

Basic Properties of Some Popular EO Materials ${ }^{11}$

\begin{tabular}{|c|c|c|c|}
\hline Material & $\begin{array}{l}\text { EO coefficient } \\
r_{i j}\left(10^{-12} \mathrm{~m} / \mathrm{V}\right)\end{array}$ & $\begin{array}{l}\text { Index of } \\
\text { refraction }\end{array}$ & $\begin{array}{l}\text { Dielectric } \\
\text { constant }\end{array}$ \\
\hline \multirow[t]{2}{*}{$\overline{\text { PLZT }}$} & $r_{13}=67$ & $n_{0}=2.312$ & \\
\hline & $r_{33}=1340$ & $n_{\mathrm{e}}=2.299$ & \\
\hline \multirow{3}{*}{$\mathrm{LiNbO}_{3}$} & $r_{13}=9.6$ & $N_{0}=2.286$ & $\varepsilon_{1}=\varepsilon_{2}=78$ \\
\hline & $r_{22}=6.8$ & $n_{\mathrm{e}}=2.200$ & $\varepsilon_{3}=32$ \\
\hline & $r_{33}=31$ & & \\
\hline \multirow[t]{2}{*}{$\mathrm{LiTaO}_{3}$} & $r_{13}=8.4$ & $n_{0}=2.176$ & $\varepsilon_{1}=\varepsilon_{2}=51$ \\
\hline & $r_{33}=30.5$ & $n_{e}=2.180$ & $\varepsilon_{3}=45$ \\
\hline \multirow[t]{2}{*}{$\mathrm{KH}_{2} \mathrm{PO}_{4}(\mathrm{KDP})$} & $r_{41}=8$ & $n_{0}=1.507$ & $\varepsilon_{1}=\varepsilon_{2}=42$ \\
\hline & $r_{63}=11$ & $n_{e}=1.467$ & $\varepsilon_{3}=21$ \\
\hline \multirow[t]{2}{*}{$\mathrm{KD}_{2} \mathrm{PO}_{4}\left(\mathrm{KD}^{*} \mathrm{P}\right)$} & $r_{63}=24.1$ & $n_{0}=1.502$ & $E_{3}=50$ \\
\hline & & $n_{e}=1.462$ & \\
\hline \multirow[t]{2}{*}{$\left(\mathrm{NH}_{4}\right) \mathrm{H}_{2} \mathrm{PO}_{4}(\mathrm{ADP})$} & $r_{41}=23.41$ & $n_{0}=1.522$ & $\varepsilon_{1}=\varepsilon_{2}=58$ \\
\hline & $r_{63}=7.83$ & $n_{e}=1.477$ & $\varepsilon_{3}=14$ \\
\hline $\mathrm{Ba}_{0.25} \mathrm{Sr}_{0.75} \mathrm{Nb}_{2} \mathrm{O}_{6}$ & $r_{13}=67$ & $n_{0}=2.3117$ & $\varepsilon_{3}=3400(15 \mathrm{MHz})$ \\
\hline \multirow[t]{2}{*}{$(\mathrm{SBN}, \mathrm{Tc}=395 \mathrm{~K})$} & $r_{43}=1340$ & $n_{e}=2.2987$ & \\
\hline & $r_{51}=42$ & & \\
\hline \multirow[t]{3}{*}{$\mathrm{KNbO}_{3}$} & $r_{13}=28 r_{23}=1.3$ & $n_{1}=2.280$ & \\
\hline & $r_{42}=380 r_{33}=64$ & $n_{2}=2.329$ & \\
\hline & $r_{51}=105$ & $n_{3}=2.169$ & \\
\hline
\end{tabular}

a All properties are measured at $633 \mathrm{~nm}$ (for optical properties), and low frequency (for dielectric constant). All properties are approximate, and should be verified with material vendors during procurement. (From Yariv, A. Optical Electronics in Modern Communications; Oxford University Press: New York, 1997. With permission.)

erty information. Extensive lists of material properties can also be found in the literature. ${ }^{33}$ Electro-optical properties of some common crystals are shown in Table 12.5.

Crystal purity, optical quality, internal strain, physical size, doping, domain structure, electrical conductivity, and other quality measures can vary widely from vendor to vendor, from one boule to the next by a single vendor, and within a single boule as well. These and related factors have led the industry to focus on a few materials that are in relatively large-scale production, such as lithium niobate and ADP, which are covered in Section 12.5.2. Other materials, such as lithium tantalate and KTP, are also covered since they are steadily increasing in quality and availability.

Materials with very high EO coefficients, such as bulk form strontium barium niobate (SBN) and PLZT in the form of deposited films, are starting to appear. Other "new" materials are doped forms of lithium niobate or tantalate, and stoichiometric lithium niobate and tantalate. Many of these materials still exhibit performance variations between suppliers-it is best to discuss your needs with several crystal suppliers and perform multiple sample runs to ensure that the proper selection is made. 


\subsubsection{ADP, KDP, and Related Isomorphs}

Relatively easy commercial growth processes make ADP (NH4H2PO4) and KDP $\left(\mathrm{KH}_{2} \mathrm{PO}_{4}\right)$ popular materials for bulk EO devices. High optical quality crystals can be grown to over $10 \mathrm{~cm}$ diameter, and can be cut, polished, and mounted without undue difficulty. In both materials, the hydrogen atoms may be replaced with deuterium. In this case they are referred to as $\mathrm{AD}^{*} \mathrm{P}$ and $\mathrm{KD}^{*} \mathrm{P}$. This replacement results in an increase in the linear $\mathrm{EO}$ coefficients by a factor of $\sim 2.5$.

The resistivity of ADP and related materials is very high, typically $>10^{10} \Omega \mathrm{cm}$ when operated near room temperature. As operating temperatures approach the Curie temperature, $C_{\mathrm{T}}$, the loss tangent and the dielectric constant increase, leading to heating and high power draws on electronic drivers if operated at high speeds. Heating in the crystal also leads to beam distortion via the thermo-optic properties of the material.

One major drawback to using ADP and its isomorphs in EO scanning systems is that the materials are hygroscopic. They are typically housed in a hermetic package that is filled with a dry gas or an index matching liquid that also provides electrical insulation. Some of the liquids are toxic, although the packaging is generally reliable.

\subsubsection{Lithium Niobate and Related Materials}

A large class of ferroelectric materials have the form $\mathrm{A}^{1+} \mathrm{B}^{5+} \mathrm{O}_{3}$ or $\mathrm{A}^{2+} \mathrm{B}^{4+} \mathrm{O}_{3}$, and are related to the mineral perovskite $\left(\mathrm{CaTiO}_{3}\right)$. Several of these materials are in mass production for devices based on their piezoelectric properties, such as $\mathrm{LiNbO}_{3}$ for surface acoustic wave filters, which are found in cell phones and a host of other signal processing applications. Czochralski growth is the typical practice, with boule diameters approaching $15 \mathrm{~cm}$ for $\mathrm{LiNbO}_{3}$, although 7.5 and $10 \mathrm{~cm}$ is more common. During the processing of crystal boules, they are typically poled to form a single domain throughout by heating to a point near the Curie temperature and then applying a DC electric field, which is maintained during the cooldown. This ensures that all crystal domains have uniform orientation-a critical consideration for good optical quality of the ensuing device.

The perovskite materials are not water soluble, eliminating some of the packaging problems encountered with ADP and similar materials. Another use of perovskites, in particular lithium tantalate, is as a pyroelectric detection element. If precautions are not taken in handling and processing, extremely high voltages can be generated between the faces of wafers. This charging can lead to electrical flashovers, which can damage electrodes or other coatings, or it can damage attached electrical equipment such as drivers or thermocouples. Wherever possible, controlled slow heating and cooling are recommended; the use of air ionizers in the work space also mitigates these effects.

The piezoelectric properties of the perovskites must be considered whenever the deflector will be operated at high speeds. The electrostrictive strain component of the index change can be as large as the EO component if a mechanical resonance is present. The mechanical performance of the entire device assembly — crystal, electrical leads, mounting adhesive and mounting base-must be considered early on, but careful testing is still a requirement.

Lithium niobate $\left(\mathrm{LiNbO}_{3}\right)$ and lithium tantalate $\left(\mathrm{LiTaO}_{3}\right)$ are the most common perovskite materials in use. For reasons of producibility and quality, they are typically grown to be slightly lithium-rich-this is referred to as congruently grown niobate or tantalate. These congruent materials exhibit Curie temperatures of 1470 and $890 \mathrm{~K}$, respectively, giving them stable EO properties at room temperature or slightly elevated temperatures. They are also commercially available with fairly consistent properties across several vendors. 
Recently, stoichiometric lithium niobate and lithium tantalate have been produced in commercially relevant sizes. . $^{18,34}$ These materials exhibit lower coercive fields, which leads to easier fabrication of poled devices, and higher EO coefficients, as well as a broader transmission range. Being relatively new, it is best to contact the crystal growers for detailed information on properties and processing practices for stoichiometric materials.

Various dopants can be introduced when growing lithium niobate or tantalate to alter properties for special applications. Magnesium is a common dopant for lithium niobate, added to mitigate photorefractive damage from short wavelengths. This variant of the material is often used when producing SHG or other nonlinear devices, typically in the visible spectrum.

Significant effort has been applied to the problem of domain inversion processing of lithium niobate and, to a lesser extent, lithium tantalate. These efforts were driven primarily by interests in SHGs and related nonlinear devices, but the practices are transferable to the production of scanners, as mentioned previously.

Barium titanate $\left(\mathrm{BaTiO}_{3}\right.$ ) and $\mathrm{KTN}$ (a solid solution of $\mathrm{KTaO}_{3}$ and $\mathrm{KNbO}_{3}$ ) belong in the perovskite group of materials. They have good EO properties, but have critical temperatures near or below room temperature. This makes some properties very temperature dependent, and can result in creating or changing ferroelectric domains simply by handling and processing the crystal. They are not in wide use at this time.

\subsubsection{Potassium Titanyl Phosphate (KTP)}

In 1976 the Du Pont Company reported ${ }^{35}$ on the growth and properties of the crystalline material $\mathrm{K}_{x} \mathrm{Rb}_{1-x} \mathrm{TiOPO}_{4}$. The material is ferroelectric and has found use in the production of SHGs and other nonlinear devices. The material is relatively difficult to grow (compared to lithium niobate), although the situation is improving due in part to military interest in the material, with slabs over $40 \mathrm{~mm}$ square being produced.

Dopants and special processing are used to produce various grades of KTP, one must check with manufacturers for availability and detailed properties. It is difficult to produce domain-inverted devices in KTP since the coercive field and dielectric strength are very near each other. Problems have also been encountered with relatively high electrical conductivity of the crystals, especially in large flux-grown crystals, further limiting its appeal.

\subsubsection{Other Materials}

\subsubsection{AB-Type Binary Compounds}

The main interest in these materials has been for EO devices in the infrared, particularly at $10.6 \mu \mathrm{m}$ for use in $\mathrm{CO}_{2}$ laser systems. GaAs, $\mathrm{ZnTe}, \mathrm{ZnS}, \mathrm{CdS}$, and CdTe are among the most common materials that are available in large sizes. The EO coefficients of these materials are relatively small, only about $10 \%$ of lithium niobate, but their transmission beyond 10 $\mu \mathrm{m}$ may make them useful in some applications, especially for military uses.

\subsubsection{Kerr Effect in Liquids}

Much attention has been paid in the past to Kerr effect liquids, especially nitrobenzene. ${ }^{1}$ The attraction was due to the high purity of materials compared to most crystals, and the basically unlimited size of the resulting device.

Advances in crystal growth techniques have largely mitigated the perceived quality and size advantages a liquid material may offer. In addition, the liquids can exhibit heating, 
currents, turbulence, and other behavior that affects performance. They are not widely used or considered for application at this time.

\subsubsection{Electro-Optic Ceramics in the $(\mathrm{Pb}, \mathrm{La})(\mathrm{Zr}, \mathrm{Ti}) \mathrm{O}_{3}$ System}

Lanthanum-modified lead zirconate titanate (PLZT) ceramic materials have been investigated since 1969 for their EO properties, which can be tailored to a degree by controlling the precise chemical makeup of the material. ${ }^{1}$

These materials are not available in large single crystal form, limiting their application to scanning systems. Scanning devices based on thin films of PLZT have been proposed, but the problems of coupling into and out of such films are daunting.

\subsubsection{Other Materials}

Significant EO materials development is still ongoing, driven by the reality that almost all current EO systems could be improved by using materials with higher EO coefficients, higher optical damage limits, lower conductivity, or improvements in other technical parameters. Efforts can be roughly characterized as either creating new materials or modifying existing ones.

An example of a relatively new material is SBN. The crystal is grown by the Czochralski method, with boule diameters typically under $50 \mathrm{~mm}$. There are several, slightly different formulations available, and quality and properties can vary even in modestly sized samples. Very high EO coefficients and relatively low Curie temperature make the material attractive for future applications. It is best to contact the material growers for current specifications prior to developing a design with an SBN element.

An example of modifications to a standard material is the development of magnesium doped lithium niobate, available from a variety of suppliers. The doping raises the optical damage threshold in the short wavelength part of the transmission band-a characteristic important for SHG devices, among others.

Careful selection, specification, inspection, and qualification of materials are a key to successful EO system design. Given the continuous improvement in crystal growing practices, inspection techniques, and materials formulation it is imperative to work closely with the materials suppliers during the design process.

\subsubsection{Material Selection}

Currently, there are only a handful of materials suitable for use in commercial EO systems. In addition to optical transparency at the desired wavelength, the following factors are common to nearly all applications.

1. High electrical resistivity: greater than $10^{10} \Omega \mathrm{cm}$ is desired. This requirement stems from the desire for no resistive heating when operating voltages (typically hundreds of volts) are applied to the device. Ion migration can also occur, especially in the presence of DC fields, which can create substantial optical perturbations.

2. High optical homogeneity: refractive index variations of less than 1 in $10^{6}$ are desired. This requirement helps to preserve beam quality, and can also be a stand-in for crystal compositional variations. 
3. Large EO effect: absolute index variations of at least $10^{-4}$ are desired, with reasonable applied voltage. Excessively high voltages create packaging problems, longterm drift due to ion migration and require high driver power when high-speed operation is required.

4. Processability: standard handling and process operations should not impact material quality or device performance. For reasonable cost, the materials must be able to be oriented, cut, polished, AR coated, and mounted with only minor (if any) departure from practices used for other optical materials.

If the device is going to be operated at high frequencies, the dielectric constant and loss tangent become important considerations, with thermal conductivity and temperature dependence of optical properties also needing to be considered.

If the device under consideration will be produced via a domain inversion process, other factors must be added to the list of considerations. Obviously it must be ferroelectric, which implies that piezoelectric and pyroelectric effects must also be taken into consideration. Also, the desire to pole the material will restrict the orientations available. Poled devices should also not be operated near to their coercive field or near the Curie temperature, which can be quite low for some materials, or there is a chance of depoling occurring.

\subsection{ELECTRO-OPTIC DEFLECTION SYSTEM DESIGN PROCESS}

Selecting a system design for a particular set of operating parameters is an iterative process, likely requiring thorough analysis of multiple trial designs. The complex interplay of material properties, EO element geometry, electronic power consumption, operating speeds, and the realities of current fabrication methods does not lend itself to a closed form solution, nor are there large catalogs of standard alternatives to choose from.

The recommended process is:

1. Verify that the speed, optical efficiency, or ruggedness of an EO deflector is required. If other technologies such as galvanometers or $\mathrm{AO}$ deflectors can be used, they are likely going to triumph in a head-to-head comparison of total system cost and complexity.

2. Consider the wavelength of the laser to be used. Few, if any, materials are optically clear across the entire spectrum of wavelengths available today. Also, many material properties vary with wavelength so it is important to look at the properties of interest at the wavelength of interest.

3. Consider whether the system can be built using a single linear polarization. If not, it is likely that beam splitting and recombining after deflection will be required, adding significant complexity and cost to the system.

4. Review the operating environment in light of safety and reliability of high-voltage electrical systems. If moisture and dust are present, the EO scanner will likely need to be placed in a sealed housing, adding length and additional windows to the optical path. 
5. Select appropriate design guidelines and safety factors such as electrical creepage distances, dielectric strength, and optical power per unit area on surfaces. These guidelines and safety factors may bound the design options.

6. Generate and analyze trial designs. One aspect that is sometimes overlooked is the mechanical response of the EO material. All attractive EO crystals exhibit piezoelectric responses to some degree. In some cases, high-speed electrical pulses can excite mechanical resonances that create a time-varying strain in the material, which can alter the deflection from that expected or contribute to beam losses.

7. Verify that the selected design can actually be built by consulting with the appropriate material suppliers, electronics designers, optical designers, and engineers familiar with current practice for all manufacturing steps.

\subsection{CONCLUSIONS}

Electro-optic scanning systems can be very fast and optically very efficient. This performance often comes at the cost of working near the cutting edge of materials, electronics, and processing technologies. The demands for speed are likely to continue increasing, however, as laser and computing technology continue to advance. To address these demands, a true systems approach should be used when designing an EO scanning system. The overlapping implications of decisions in areas as diverse as HV electronic drivers, mechanical isolation, temperature control, and beam size must be weighed carefully.

Large scale application of EO scanners has not yet occurred due to the difficulties and uncertainties discussed in this chapter. Recent advances in telecommunications switching, computer-to-plate printing, and biomédical imaging applications are driving development of EO devices and electronic drivers at a rapid pace. New EO materials, with new combinations of properties, are also being developed. Such progress in each of the key areas of EO scanning system design and construction may eventually lead to their wider application.

\section{ACKNOWLEDGMENTS}

Several people contributed ideas, commentary and references during the preparation of this section. Of particular note is Richard Stolzenberger, PhD, now a consultant. The authors are also grateful for the support (and tolerance) of our spouses. Gerald F. Marshall, the volume editor, also provided the ongoing encouragement to complete the effort in the face of industry turmoil.

\section{REFERENCES}

1. Ireland, C; Ley, J. Electrooptical scanners. In Optical Scannin. Marcel Dekker: New York, 1987; 687-778.

2. Stancil, D.D. Electro-optical scanners. In Encyclopedia of Optical Engineering. Marcel Dekker: New York, 2003; 456-474. 
3. Lee, S.M.; Hauser, S.M. Kerr constant evaluation of organic liquids and solutions. Rev. Sei. Instruments 1964, 35, 1679.

4. Kruger, R.; Pepperl, R.; Schmidt, U. Electrooptic materials for digital light beam deflectors. Proc. IEEE 1973, 61, 992.

5. Chiu, Y.; Zou, J.; Stancil, D.D.; Schlesinger, T.E. Shape-optimized electrooptic beam scanners: Analysis, design, and simulation. J. Lightwave Technol. 1999, 17(1), 108-114.

6. Lotspeich, J.F. Electrooptic light-beam deflection. IEEE Spectrum 1968, 5, February, 45-52.

7. Lee, T.C.; Zook, J.D. Light beam deflection with electrooptic prisms. IEEE J. Quantum Electronics 1968, QE-4(7), 442-454.

8. Fowler, V.J.; Buhrer, CF.; Bloom, L.R. Electro-optic light beam deflector. Proc. IEEE 1964, 52(2), 193-194.

9. Fowler, V.J.; Schlafer, J.A. Survey of laser beam deflection techniques. Appl. Optics 1966, 5(10), $1675-1682$.

10. Kiyatkin, R.P. Analysis of control field in quadrupole optical-radiation deflectors. Opt. Spectrosc. 1975, 38(2), 209-210.

11. QuickField, for finite element calculations. Retrieved from http://www.quickfield.com March 22, 2004.

12. Ireland, C; Ley, J. Electrooptical scanners. In Optical Scanning; Marshall, G., Ed.; Marcel Dekker: New York, 1987; 752-754.

13. Armstrong, J.A.; Bloembergen, N.; Ducuing, J.; Pershan, P.S. Interactions between light waves in a nonlinear dielectric. Phys. Rev. 1962, 127, 1918-1939.

14. Fejer, M.M.; Magel, G.A.; Jundt, D.H.; Byer, R.L. 'Quasi-phase-matched second harmonic generation: tuning and tolerances.' IEEE J. Quantum Electronics 1992, 28(11), 2631-2654.

15. Mizuuchi, K.; Yamamoto, K. Highly efficient quasiphase-matched 2nd harmonic generation using 1st-order periodically domain-inverted $\mathrm{LiTaO}_{3}$ waveguide. Appl. Phys. Lett. 1992, 60(11), 1283-1285.

16. Wang, Y.; Petrov, V.; Ding, Y.J.; Zheng, Y.; Khurgin, J.B.; Risk, W.P. Ultrafast generation of blue light by efficient second-harmonic generation in periodically-poled bulk and waveguide potassium titanyl phosphate. Appl. Phys. Lett. 1998, 73(7), 873-875.

17. Ktaoka, Y.; Narumi, K.; Mizuuchi, K. Waveguide-type SHG blue laser for high-density optical disk system. Rev. Laser Eng. 1998, 26(3), 256-260.

18. Gopalan, V.; Sanford, N.A.; Aust, J.A.; Kitamura, K.; Furukawa, Y. Crystal growth, characterization, and domain studies in lithium niobate and lithium tantalate ferroelectrics. In Handbook of Advanced Electronic and Photonic Materials and Devices, Nalwa, H.S., Ed.; Academic Press: New York, 2001; Vol. 4, Ferroelectrics and Dielectrics, 57-114.

19. Li, J.; Cheng, H.C.; Kawas, M.J.; Lambeth, D.N.; Schlesinger, T.E.; Stancil, D.D. Electrooptic wafer beam deflector in $\mathrm{LiTaO}_{3}$. IEEE Photonics Tech. Letts. 1996, 8(11), 1486-1488.

20. Chen, Q.; Chiu, Y.; Lambeth, D.N.; Schlesinger, T.E.; Stancil, D.D. Guided-wave electro-optic beam deflector using domain reversal in $\mathrm{LiTaO}_{3}$. J. Lightwave Technology 1994, 12(4), 1401-1404.

21. Chen, Q.; Chiu, Y.; Devasahayam, A.J.; Seigler, M.A.; Lambeth, D.N.; Schlesinger, T.E.; Stancil, D.D. Waveguide optical scanner with increased deflection sensitivity for optical data storage. In SPIE Proc. Series, Vol. 2338, 1994; Topical Meeting on Optical Data Storage, Dana Point, CA; May 16-18, 1994; 262-267.

22. Fang, J.C.; Kawas, M.J.; Zou, J.; Gopalan, V.; Schlesinger, T.E.; Stancil, D.D. Shape-optimized electrooptic beam scanners: experiment. IEEE Photonics Technol. Lett. 1999, 11(1), 66-68.

23. Chiu, Y.; Burton, R.S.; Stancil, D.D.; Schlesinger, T.E. Design and simulation of waveguide electrooptic beam deflectors. J. Lightwave Technol. 1995, 13(10), 2049-2052.

24. Feit, M.D.; Fleck, J.A., Jr. Light propagation in graded-index optical fibers. Appl. Opt. 1978, 17(24), 3990-3998.

25. Eason, R.; Boyland, A.; Mailis, S.; Smith, P.G.R. Electro-optically controlled beam deflection for grazing incidence geometry on a domain-engineered interface in $\mathrm{LiNbO}_{3}$. Optics Commun. 2001, 197, 201-207. 
26. Gnewuch, H.; Pannell, C; Ross, G.; Smith, P.G.R.; Geiger, H. Nanosecond response of Bragg deflectors in periodically poled $\mathrm{LiNbO}_{3}$. IEEE Photonics Technol. Lett. 1998, 10(12), 1730-1732.

27. Kawas, M.J. Design and characterization of domain inverted electro-optic lens stacks on $\mathrm{LiTaO}_{3}$. Department of Electrical and Computer Engineering; Carnegie Mellon University, 1996; M.S. Thesis.

28. Kawas, M.J.; Stancil, D.D.; Schlesinger, T.E. Electrooptic lens Stacks on $\mathrm{LiTaO}_{3}$ by domain inversion. J. Lightwave Technol. 1997, 15(9), 1716-1719.

29. Gahagan, K.T.; Gopalan, V.; Robinson, J.M.; Jia, Q.; Mitchell, T.E.; Kawas, M.J.; Schlesinger, T.E.; Stancil, D.D. Integrated electro-optic lens/scanner in a $\mathrm{LiTaO}_{3}$ single crystal. Appl. Optics 1999, 38(4), 1186-1190.

30. Gopalan, V.; Kawas, M.J.; Gupta, M.C.; Schlesinger, T.E.; Stancil, D.D. Integrated quasi-phasematched second-harmonic generator and electrooptic scanner on $\mathrm{LiTaO}_{3}$ single crystals. IEEE Photonics Technology Lett. 1996, 8 (12), 1704-1706.

31. Chiu, Y.; Gopalan, V.; Kawas, M.J.; Schlesinger, T.E.; Stancil, D.D.; Risk, W.P. Integrated optical device with second-harmonic generator, electrooptic lens, and electrooptic scanner in $\mathrm{LiTaO}_{3}$. J. Lightwave Technol. 1999, 17(3), 462-465.

32. Cleland, A.; Gass, H. Energy recirculating driver for capacitive load. Patent Cooperation Treaty application, document \#WO 02/14932, August 16, 2001; revised February 21, 2002.

33. Yariv, A. Optical Electronics in Modern Communications; Oxford University Press: New York, 1997.

34. Furukawa, Y.; Kitamura, K.; Suzuki, E.; Niwa, K.J. Stoichiometric $\mathrm{LiTaO}_{3}$ single crystal growth by double crucible Czochralski method using automatic powder supply system. Crystal Growth $1999,197,889$.

35. Zumsteg, F.; Bierlein, J.; Gier, T. $\mathrm{K}_{x} \mathrm{Rb}_{1-x} \mathrm{TiOPO}_{4}$ : A new nonlinear optical material. J. Appl. Phys. $1976,47,4980$.

36. Revelli, J.F. High-resolution electrooptic surface prism waveguide deflector: An analysis. Appl. Optics 1980, 19, 389-397.

37. Lee, C.L.; Lee, J.F.; Huang, J.Y. Linear phase shift electrodes for the planar electrooptic prism deflector. Appl. Optics 1980, 19, 2902-2905.

38. Sasaki, H.; De La Rue, R.M. Electro-optic multichannel waveguide deflector. Electronics Letts. 1977, 13(10), 295-296.

39. Chiu, Y.; Burton, R.S.; Stancil, D.D.; Schlesinger, T.E. Design and simulation of waveguide electrooptic beam deflectors. J. Lightwave Technol. 1995, 13(10), 2049-2052.

40. Takizawa, K. Electrooptic Fresnel lens-scanner with an array of channel waveguides. Appl. Optics 1983, 22(16), 2468-2473. 